\title{
Noncommutative quadric surfaces
}

\author{
S. Paul Smith and M. Van den Bergh*
}

\begin{abstract}
The 4-dimensional Sklyanin algebra is the homogeneous coordinate ring of a noncommutative analogue of projective 3-space. The degree-two component of the algebra contains a 2-dimensional subspace of central elements. The zero loci of those central elements, except 0 , form a pencil of noncommutative quadric surfaces. We show that the behavior of this pencil is similar to that of a generic pencil of quadrics in the commutative projective 3space. There are exactly four singular quadrics in the pencil. The singular and non-singular quadrics are characterized by whether they have one or two rulings by noncommutative lines. The Picard groups of the smooth quadrics are free abelian of rank two. The alternating sum of dimensions of Ext allows us to define an intersection pairing on the Picard group of the smooth ntive quadrics. A surprise is that a smooth noncommutative quadric can sometimes contain a "curve" having self-intersection number -2 . Many of the methods used in our paper are noncommutative versions of methods developed by Buchweitz, Eisenbud and Herzog: in particular, the correspondence between the geometry of a quadric hypersurface and maximal Cohen-Macaulay modules over its homogeneous coordinate ring plays a key role. An important aspect of our work is to introduce definitions of noncommutative analogues of the familiar commutative terms used in this abstract. We expect the ideas we develop here for 2-dimensional noncommutative quadric hypersurfaces will apply to higher dimensional noncommutative quadric hypersurfaces and we develop them in sufficient generality to make such applications possible.
\end{abstract}

Mathematics Subject Classification (2010). 14A22.

Keywords. Noncommutative algebraic geometry, noncommutative quadric surfaces, Sklyanin algebra.

\section{Contents}

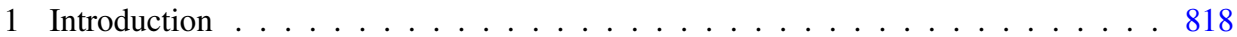

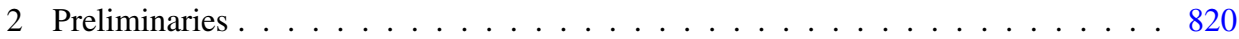

3 Maximal Cohen-Macaulay modules . . . . . . . . . . . . . . . . 824

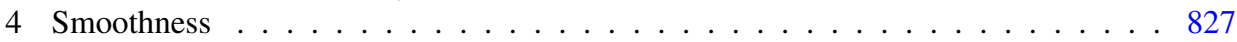

5 Quadrics in quantum $\mathbb{P}^{3} \mathrm{~s} \ldots \ldots \ldots \ldots$. . . . . . . . . . . . . . . . . . . . . . . . . . . . . .

6 The Auslander property . . . . . . . . . . . . . . . . . . . . . . . . . 835

*Paul Smith was supported by the National Science Foundation under Grants DMS-0070560 and DMS-024572. M. Van den Bergh is a director of research at the FWO. 


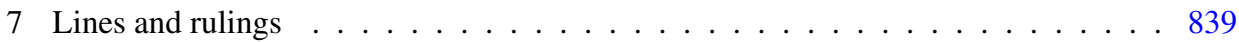

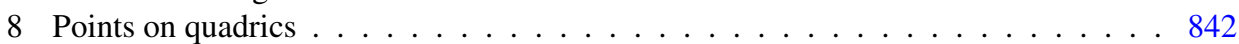

9 The Grothendieck group of a smooth quadric . . . . . . . . . . . . . . . . . . . 845

10 The Sklyanin quadrics . . . . . . . . . . . . . . . . . . . . 851

References . . . . . . . . . . . . . . . . . . . 855

\section{Introduction}

1.1. Many noncommutative analogues of $\mathbb{P}^{3}$ contain noncommutative quadric hypersurfaces. This paper studies these noncommutative quadrics and the consequences of their existence for the ambient noncommutative $\mathbb{P}^{3}$.

For example, we establish a simple "geometric" criterion for recognizing when a noncommutative quadric surface is smooth: it is smooth if and only if it has two rulings. Of course, a key point is to define the terms.

The smoothness result allows us to make further comparisons with the commutative case. For example, a generic pencil of quadrics in $\mathbb{P}^{3}$ has exactly four singular members and we show the same is true for the pencil of noncommutative quadrics in the Sklyanin analogue of $\mathbb{P}^{3}$.

1.2. A noncommutative quadric surface $Q$ is defined implicitly by defining a Grothendieck category Qcoh $Q$ that plays the role of quasi-coherent sheaves on it. We say that $Q$ is smooth of dimension two if $\operatorname{Ext}_{Q}^{3}(-,-)$ vanishes everywhere on $Q \operatorname{coh} Q$ but $\operatorname{Ext}_{Q}^{2}(-,-)$ does not.

Deciding whether a commutative variety is smooth is a local problem: one examines the local rings at its points. One can also use the Jacobian criterion on affine patches. Deciding whether a noncommutative variety is smooth is a different kind of problem because the variety can have few closed points, sometimes none at all. One cannot check smoothness by checking the homological properties of individual points. In this sense, smoothness is not a local property and global methods must be used. In particular, there is no analogue of the Jacobian criterion and singular noncommutative quadrics need not have a singular point.

Theorem 5.6 shows that the smoothness of a quadric hypersurface in a noncommutative $\mathbb{P}^{3}$ (where these terms have to be defined appropriately) is equivalent to the semisimplicity of a certain finite dimensional algebra.

1.3. We will define noncommutative quadric surfaces as degree two hypersurfaces in suitable noncommutative analogues of $\mathbb{P}^{3}$, the latter being a noncommutative space of the form Proj $S$ where $S$ is a not-necessarily-commutative connected graded ring having properties like those of the commutative polynomial ring in four variables (see Section 2.8 for a precise definition). Thus $Q=\operatorname{Proj} A$ where $A=S /(z)$ and $z \in S_{2}$ is a central regular element. (If $z$ were a normal regular element we could replace $S$ by a suitable Zhang twist in which $z$ becomes central, so there is no loss 
of generality in assuming $z$ is central.) Amongst other things, $S$ is required to be a Koszul algebra and this implies that $S /(z)$ is also Koszul.

1.4. Let $S$ denote a 4-dimensional Sklyanin algebra [14], [15], [18], [22], [23]. In this case we write

$$
\mathbb{P}_{\text {Skly }}^{3}=\text { Proj } S \text {. }
$$

The common zero locus of the two linearly independent degree-two central elements in $S$ is commutative elliptic curve $E$. The zero loci of linear combinations of these two central elements form a pencil of noncommutative quadrics $Q=\operatorname{Proj} S /(z) \subset \mathbb{P}_{\text {Skly }}^{3}$. Exactly four of these noncommutative quadrics are singular (Theorem 10.2). The base locus of the pencil is $E$. This is a direct analogue of the commutative case: the base locus of a generic pencil of quadrics in $\mathbb{P}^{3}$ is a quartic elliptic curve, and exactly four members of that pencil are singular.

1.5. The method we use to understand these noncommutative quadrics follows that of Buchweitz, Eisenbud, and Herzog in their paper [5] on maximal Cohen-Macaulay modules over quadrics, and especially the approach in the appendix of their paper. As is well known, a quadric hypersurface is smooth if and only if the even Clifford algebra determined by its defining equation is semisimple. The results in [5] establish a duality between the maximal Cohen-Macaulay modules over the coordinate ring of the quadric and the derived category of the Clifford algebra. We associate to our noncommutative quadrics $Q=\operatorname{Proj} A$ finite dimensional algebras $C$ that are analogues of even Clifford algebras and establish the "same" duality.

1.6. Let $A^{!}$be the quadratic dual of $A$. Because $A$ is a hypersurface ring, $\operatorname{Proj} A^{!}$ is an affine space. The algebra $C$ is a coordinate ring of this space in the sense that Qcoh $\left(\operatorname{Proj} A^{!}\right) \cong \operatorname{Mod} C$. We show that $Q$ is smooth if and only if $C$ is semisimple if and only if there are two distinct noncommutative "rulings" on $Q$. We show that the "lines" on $Q$ determine 2-dimensional simple $C$-modules; because the dimension of $C$ is 8 , it is semisimple if it has two non-isomorphic 2-dimensional simple modules. The method by which we associate a $C$-module to a line on $Q$ uses the fact that $A$ is a Koszul algebra, and that the lines on $Q$ determine graded maximal Cohen-Macaulay $A$-modules.

1.7. Although quantum $\mathbb{P}^{2} \mathrm{~s}$ have been classified and are well-understood in some regards, the same is not true for quantum $\mathbb{P}^{3} \mathrm{~s}$. The results in this paper are a step towards gaining a similar understanding of another class of noncommutative surfaces. In Sections 7 and 8 we obtain good information about the points and lines on such surfaces.

Furthermore, if the noncommutative quadric $Q$ is smooth there is an isomorphism $K_{0}(Q) \cong K_{0}\left(\mathbb{P}^{1} \times \mathbb{P}^{1}\right)$ of Grothendieck groups that is compatible with the Euler forms $(-,-)=\sum(-1)^{i} \operatorname{dim} \operatorname{Ext}_{Q}^{i}(-,-)$. More interestingly, the effective cones for 
$Q$ and $\mathbb{P}^{1} \times \mathbb{P}^{1}$ need not match up under this isomorphism: sometimes $Q$ contains, in effect, a-2-curve.

All unexplained terminology for noncommutative spaces can be found in either [17] or [25].

\section{Preliminaries}

Throughout $k$ denotes a field and A denotes a two-sided noetherian connected graded $k$-algebra.

The Hilbert series of a graded $k$-vector $V$ having finite dimensional components is the formal series

$$
H_{V}(t):=\sum_{n}\left(\operatorname{dim}_{k} V_{n}\right) t^{n}
$$

2.1. Graded modules. The category of graded right $A$-modules with degree zero module homomorphisms is denoted by $\operatorname{Gr} A$ and $\operatorname{gr} A$ is the full subcategory of $\mathrm{Gr} A$ consisting of noetherian modules. We write $\mathrm{D}^{\mathrm{b}}(\operatorname{gr} A)$, or just $\mathrm{D}^{\mathrm{b}}(A)$, for the associated bounded derived category.

We write $\operatorname{Ext}_{\mathrm{Gr} A}^{i}(M, N)$ for the extension groups in $\mathrm{Gr} A$, and define

$$
\underline{\operatorname{Ext}}_{A}^{*}(M, N):=\bigoplus_{i \in \mathbb{Z}} \operatorname{Ext}_{\mathrm{Gr} A}^{i}(M, N(i)) .
$$

2.2. Syzygies. When $M \in \operatorname{gr} A$ we write $\Omega^{i} M$ for the $i^{\text {th }}$ syzygy in $\operatorname{gr} A$ obtained from a minimal graded resolution of $M$. Since $A$ is connected graded, $\underline{\operatorname{Ext}}_{A}^{i}\left(\Omega^{d} M, k\right) \cong \underline{\operatorname{Ext}}_{A}^{i+d}(M, k)$ for all $i \geq 0$. We often write $\Omega M$ for $\Omega^{1} M$.

2.3. Linear resolutions. An $M$ in gr $A$ has a linear resolution if for all $i$ the $i^{\text {th }}$ term in its minimal projective resolution is a direct sum of copies of $A(-i)$ or, equivalently, if $\underline{\operatorname{Ext}}_{A}^{i}(M, k)_{j}=0$ whenever $i+j \neq 0$. We write $\operatorname{Lin}(A)$ for the full subcategory of gr $A$ consisting of modules having a linear resolution; $\operatorname{Lin}(A)$ is closed under direct summands and extensions:

If $M \in \operatorname{Lin}(A)$, then $\left(\Omega^{n} M\right)(n) \in \operatorname{Lin}(A)$ too.

If $M \in \operatorname{Lin}(A)$, then $H_{\underline{\operatorname{Ext}}_{A}^{*}(M, k)}(t) H_{A}(-t)=H_{M}(-t)$.

2.4. Koszul duality. See [3] for basic information about Koszul algebras.

Let $A$ be a connected Koszul algebra and $A^{!}$its quadratic dual.

The Koszul property says that the natural homomorphism $A^{!} \rightarrow \underline{\operatorname{Ext}}_{A}^{*}(k, k)$ is an isomorphism of graded $k$-algebras. If $M$ is a graded $A$-module, the Yoneda product makes $\underline{\operatorname{Ext}}_{A}^{*}(M, k)$ a graded left $A^{!}$-module with degree $i$ component $\underline{\operatorname{Ext}}_{A}^{i}(M, k)$. 
A graded $A$-module $M$ is stably linear if $M_{\geq n}(n)$ has a linear resolution for $n \gg 0$. We write $\mathrm{D}_{\mathrm{sl}}^{\mathrm{b}}(A)$ for the full subcategory of $\mathrm{D}^{\mathrm{b}}(A)$ of complexes having stably linear homology.

By [20] there is a duality

$$
K: \mathrm{D}_{\mathrm{sl}}^{\mathrm{b}}(A) \rightarrow \mathrm{D}_{\mathrm{sl}}^{\mathrm{b}}\left(A^{!}\right)
$$

given by

$$
K M=T\left(\underline{\operatorname{Hom}}_{A}(M, k)\right),
$$

where $T$ is the re-grading functor

$$
(T V)_{j}^{i}=V_{-j}^{i+j}
$$

where the upper index is the homological degree and the lower index the grading degree. The duality $K$ restricts to a duality

$$
\operatorname{Lin}(A) \rightarrow \operatorname{Lin}\left(A^{!}\right), \quad M \mapsto \underline{\operatorname{Ext}}_{A}^{*}(M, k),
$$

with degree $i$ component $\operatorname{Ext}_{A}^{i}(M, k)$. The Koszul duality functor $K$ satisfies

$$
K(M[1]) \cong(K M)[-1] \text { and } K(M(1)) \cong(K M)[-1](1) .
$$

Theorem 2.1 (Jørgensen [8], Thm. 3.1). Let A be a two-sided noetherian, connected, graded $k$-algebra that is Koszul and has a balanced dualizing complex [24], [26]. Then every finitely generated A-module is stably linear. Thus

$$
\mathrm{D}_{\mathrm{sl}}^{\mathrm{b}}(A)=\mathrm{D}^{\mathrm{b}}(A)
$$

2.5. Cohen-Macaulay rings and modules. Let $A$ be a right and left noetherian, connected, graded $k$-algebra having a balanced dualizing complex $R \cdot$ [24], [26].

We say that $A$ is Cohen-Macaulay of depth $d$ if there is an $A$ - $A$-bimodule $\omega_{A}$ such that $R^{\cdot} \cong \omega_{A}[d]$. We call $\omega_{A}$ the dualizing module for $A$. By [2], Prop. 7.9, $\omega_{A}$ is finitely generated on each side. We say $A$ is Gorenstein if it is Cohen-Macaulay and $\omega_{A}$ is an invertible bimodule. This is equivalent to the requirement that $\omega_{A}$ is isomorphic to $A(\ell)$ for some $\ell$ as both a right and as a left module.

The local cohomology functors

$$
H_{\mathfrak{m}}^{i}(-)=\lim _{\longrightarrow} \underline{\operatorname{Ext}}_{A}^{i}\left(A / A_{\geq n},-\right)
$$

are defined on graded right $A$-modules. Here $\mathfrak{m}$ denotes the maximal ideal $A_{\geq 1}$. We write $H_{\mathrm{m}}^{i}$ 。 for the local cohomology modules for left modules. The depth of an $A$-module $M$ is the smallest integer $i$ such that $H_{\mathfrak{m}}^{i}(M) \neq 0$. A finitely generated module $M$ is Cohen-Macaulay if either $M=0$ or only one $H_{\mathfrak{m}}^{i}(M)$ is non-zero. For the rest of this section we assume that $A$ is Cohen-Macaulay of depth $d$ in the 
sense of the previous paragraph. Then $A$ is a Cohen-Macaulay $A$-module of depth $d$ in the sense of the present paragraph. Furthermore, there is an isomorphism

$$
\omega_{A} \cong H_{\mathfrak{m}}^{d}(A)^{*}
$$

of $A$ - $A$-bimodules and, for every $M \in \bmod A$,

$$
\underline{\operatorname{Ext}}_{A}^{i}\left(M, \omega_{A}\right) \cong H_{\mathfrak{m}}^{d-i}(M)^{*}
$$

as graded left $A$-modules [26], Thm. 4.2.

2.6. The condition $\chi$. Let $A$ be a connected graded $k$-algebra.

We say $A$ satisfies condition $\chi$ if $\underline{\operatorname{Ext}}_{A}^{i}(k, M)$ is finite dimensional for all finitely generated $M$ and all $i$. By [2], Cor. 3.6, this is equivalent to $H_{\mathfrak{m}}^{i}(M)$ being zero in large positive degree for all $i$ and all finitely generated $M$. Hence, if $A$ is noetherian and Cohen-Macaulay, formula (2.1) implies that $A$ satisfies $\chi$ on both sides [26], Thm. 4.2. The precise relationship between condition $\chi$ and the Cohen-Macaulay property is given by [24], Thm. 6.3.

A noetherian, connected, graded algebra $A$ satisfying $\chi$ has finite depth, and for every $M \in \bmod A$ of finite projective dimension,

$$
\operatorname{pdim} M+\operatorname{depth} M=\operatorname{depth} A \text {. }
$$

As in the commutative case we call this the Auslander-Buchsbaum formula. The noncommutative version was proved by Jørgensen [7].

2.7. Noncommutative spaces. Let $A$ be a connected graded noetherian $k$-algebra.

Artin and Zhang [2] define Proj $A$ to be the (imaginary) noncommutative scheme defined implicitly by declaring that the category of "quasi-coherent sheaves" on it is

$$
\mathrm{Q} \operatorname{coh}(\operatorname{Proj} A):=\mathrm{QGr} A:=\frac{\operatorname{Gr} A}{\operatorname{Fdim} A}
$$

where Fdim $A$ is the full subcategory consisting of direct limits of finite dimensional modules. We write $\pi: \operatorname{Gr} A \rightarrow \mathrm{Q} \operatorname{coh}(\operatorname{Proj} A$ ) for the quotient functor and $\omega$ for its right adjoint. Modules in Fdim $A$ are said to be torsion.

We also define

$$
\operatorname{coh}(\operatorname{Proj} A):=\operatorname{qgr} A:=\frac{\operatorname{gr} A}{\operatorname{Fdim} A \cap \operatorname{gr} A} .
$$

It is the full subcategory of noetherian objects in Qcoh(Proj $A$ ).

Write $X=\operatorname{Proj} A$ and $\mathcal{O}_{X}=\pi A$.

Artin and Zhang define the cohomology groups

$$
H^{q}(X, \mathcal{F}):=\operatorname{Ext}_{X}^{q}\left(\mathcal{O}_{X}, \mathcal{F}\right) .
$$


If $M$ is a graded $A$-module, there is an exact sequence

$$
0 \rightarrow H_{\mathfrak{m}}^{0}(M) \rightarrow M \rightarrow \omega \pi M \rightarrow H_{\mathfrak{m}}^{1}(M) \rightarrow 0,
$$

and, if $\mathcal{M}=\pi M$, then

$$
H^{q}(X, \mathcal{M}) \cong H_{\mathfrak{m}}^{q+1}(M)_{0}
$$

for $q \geq 1$ [2], Prop. 7.2.

Following [26], Defn. 2.4, we say that $X$ is Cohen-Macaulay of dimension $d$ if there exists $\omega_{X} \in \operatorname{coh} X$ and isomorphisms

$$
H^{q}(X,-) \rightarrow \operatorname{Ext}_{X}^{d-q}\left(-, \omega_{X}\right)^{*}
$$

on $\operatorname{coh} X$ for all $q$.

Suppose that $A$ is noetherian and Cohen-Macaulay of depth $d+1$. Since $A$ satisfies $\chi,(2.3)$ allows us to quote [26], Thm. 2.3, which says that $X=\operatorname{Proj} A$ is Cohen-Macaulay of dimension $d$ with $\omega_{X} \cong \pi\left(H_{\mathfrak{m}}^{d+1}(A)^{*}\right)$.

2.8. Noncommutative analogues of $\mathbb{P}^{\boldsymbol{n}}$. The quadric surfaces of interest to us are degree two hypersurfaces in quantum $\mathbb{P}^{3} \mathrm{~S}$.

For the purposes of this paper a quantum $\mathbb{P}^{n}$ is a noncommutative scheme Proj $S$ for which $S$ is a connected graded $k$-algebra with the following properties:

(1) $S$ has global homological dimension $n+1$ on both sides and

$$
\operatorname{Ext}^{i}(k, S) \cong \begin{cases}0 & \text { if } i \neq n+1 \\ k & \text { if } i=n+1\end{cases}
$$

for the right and left trivial modules $k=S / S_{\geq 1}$ (i.e., $S$ is an Artin-Schelter (AS) regular algebra);

(2) $S$ is right and left noetherian;

(3) $H_{S}(t)=(1-t)^{-n-1}$.

J. J. Zhang showed that these conditions imply that $S$ is a Koszul algebra and has dualizing module $\omega_{S} \cong A(-n-1)$ [16], Thm. 5.11. Furthermore, $S$ satisfies $\chi$ on both sides. When $n+1 \leq 4, S$ is a domain by [1].

A result of Shelton and Vancliff [13], Lemma 1.3, shows that for quantum $\mathbb{P}^{3} \mathrm{~s}$ the hypotheses (1)-(3) are not the most efficient - one can slightly weaken them.

Write $\mathbb{P}_{n c}^{n}=\operatorname{Proj} S$. The hypotheses ensure that $H^{n+1}\left(\mathbb{P}_{n c}^{n},-\right)=0$ and that the dimensions of $H^{q}\left(\mathbb{P}_{n c}^{n}, \mathcal{O}(r)\right)$ agree with those in the commutative case.

The Grothendieck group of a quantum $\mathbb{P}^{n}$ is isomorphic to $\mathbb{Z}\left[t, t^{-1}\right] /(1-t)^{n+1}$ with $[\mathcal{F}(-1)]=[\mathcal{F}] t$. There is a good notion of degree for closed subspaces of Proj $S$. In particular, if $z \in S$ is a homogeneous normal element, meaning that $S z=$ $z S$, then Proj $S /(z)$ is a hypersurface of degree equal to $\operatorname{deg} z$. Write $A=S /(z)$. Then $A$ is Gorenstein of depth $n$, and satisfies $\chi$. In particular, $H^{n}(\operatorname{Proj} A,-)=0$. 
Lemma 2.2. If $S$ is a connected graded $k$-algebra of finite global dimension and $H_{S}(t)=(1-t)^{-n}$, then the Hilbert series of every finitely generated A-module of GK-dimension one is eventually constant.

Proof. The minimal projective resolution of a finitely generated $A$-module $M$ is finite, and all terms are direct sums of shifts of $A$, so the Hilbert series of the module is of the form $f(t)(1-t)^{-n}$ for some $f(t) \in \mathbb{Z}\left[t, t^{-1}\right]$. The hypothesis on the GKdimension means that we can rewrite this as $g(t)(1-t)^{-1}$ with $g(t) \in \mathbb{Z}\left[t, t^{-1}\right]$. Hence $\operatorname{dim} M_{n}=g(1)$ for $n \gg 0$.

\section{Maximal Cohen-Macaulay modules}

Suppose that $A$ is a connected, graded, noetherian, and Cohen-Macaulay of depth $d \geq 1$.

3.1. A noetherian $A$-module $M$ is maximal Cohen-Macaulay if depth $M=d$. We write $\operatorname{MCM}(A)$ for the full subcategory of gr $A$ consisting of the maximal CohenMacaulay modules; we consider the zero module to be maximal Cohen-Macaulay, so $\operatorname{MCM}(A)$ is an additive category.

If $i \geq d$, then $\Omega^{i} M \in \operatorname{MCM}(A)$ for all $M \in \operatorname{gr} A$. If $M$ is in $\operatorname{MCM}(A)$ so is $\Omega M$.

The stable category of maximal Cohen-Macaulay modules, denoted by $\operatorname{MCM}(A)$, has the same objects as $\operatorname{MCM}(A)$ and morphisms

$$
\operatorname{Hom}_{\underline{\underline{M C M}}(A)}(M, N):=\frac{\operatorname{Hom}_{\mathrm{Gr} A}(M, N)}{P(M, N)}
$$

where $P(M, N)$ consists of the degree zero $A$-module maps $f: M \rightarrow N$ that factor through a projective in $\mathrm{Gr} A$.

3.2. The next two results are due to Buchweitz and are stated in his appendix to the paper [5]; see also [4].

Theorem 3.1. Suppose that $A$ is Gorenstein. Then $\underline{\operatorname{MCM}}(A)$ is a triangulated category with respect to the translation functor $M[-1]:=\Omega M$. If $M$ and $N$ are maximal Cohen-Macaulay modules, then

$$
\operatorname{Hom}_{\underline{\mathrm{MCM}}(A)}(M, N[n]) \cong \operatorname{Ext}_{\mathrm{Gr} A}^{n}(M, N)
$$

for all $n \geq 1$. 
Theorem 3.2. Let A be a Gorenstein, connected, graded, Koszul algebra over a field $k$, and $A^{!}$its quadratic dual. The Koszul duality functor $K$ fits into a commutative diagram

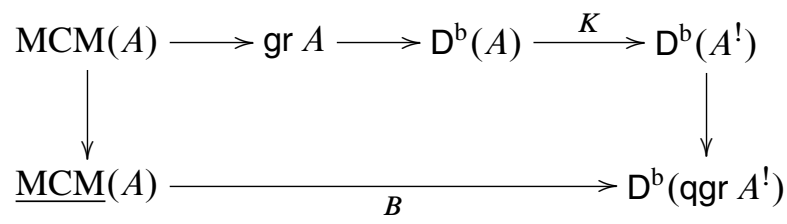

in which the bottom arrow is a duality

$$
\underline{\operatorname{MCM}}(A) \cong \mathrm{D}^{\mathrm{b}}\left(\operatorname{qgr} A^{!}\right), \quad M \mapsto \underline{\operatorname{Rom}}_{A}(M, k) .
$$

The $t$-structure on $\underline{\operatorname{MCM}}(A)$ induced by the natural $t$-structure on $\mathrm{D}^{\mathrm{b}}\left(\mathrm{qgr} A^{!}\right)$is

$$
\begin{aligned}
& \underline{\operatorname{MCM}}(A)^{\geq p}=\left\{M \mid \underline{\operatorname{Ext}}_{A}^{i}(M, k)_{j}=0 \text { for } i+j>p\right\}, \\
& \underline{\operatorname{MCM}}(A)^{\leq p}=\left\{M \mid \underline{\left.\operatorname{Ext}_{A}^{i}(M, k)_{j}=0 \text { for } i+j<p\right\} .}\right.
\end{aligned}
$$

The heart for this $t$-structure consists of the maximal Cohen-Macaulay modules having a linear resolution.

We will refer to the duality

$$
B: \underline{\operatorname{MCM}}(A) \rightarrow \mathrm{D}^{\mathrm{b}}\left(\mathrm{qgr} A^{!}\right)
$$

in Theorem 3.2 as "Buchweitz's duality".

Lemma 3.3. Suppose that $A$ is a connected graded, Gorenstein, Koszul algebra. Write $F$ for the composition

$$
\operatorname{gr} A \rightarrow \mathrm{D}^{\mathrm{b}}(A) \stackrel{K}{\longrightarrow} \mathrm{D}^{\mathrm{b}}\left(A^{!}\right) \rightarrow \mathrm{D}^{\mathrm{b}}\left(\mathrm{qgr} A^{!}\right) .
$$

If $M \in \operatorname{gr} A$, then $F(\Omega M) \cong(F M)[1]$ and $F(\Omega M(1)) \cong(F M)(1)$.

Proof. There is an exact sequence $0 \rightarrow \Omega M \rightarrow \bigoplus_{i \in I} A(i) \rightarrow M \rightarrow 0$ for some multiset $I$, and hence a distinguished triangle $\Omega M \rightarrow \bigoplus_{i \in I} A(i) \rightarrow M \rightarrow$ in $\mathrm{D}^{\mathrm{b}}(A)$. The image of $K(\oplus A(i))$ in $\mathrm{D}^{\mathrm{b}}\left(\mathrm{qgr} A^{!}\right)$is zero, so there is an isomorphism

$$
\pi K M \stackrel{\sim}{\longrightarrow} \pi K((\Omega M)[1]) \cong \pi K(\Omega M)[-1]
$$

in $\mathrm{D}^{\mathrm{b}}\left(\operatorname{qgr} A^{!}\right)$. Hence $F M \cong F(\Omega M)[-1]$.

The other isomorphism is established in a similar way.

Lemma 3.4. Suppose that $A$ is a connected graded, Gorenstein algebra. The isomorphism classes of indecomposable objects in $\underline{\mathrm{MCM}}(A)$ are in bijection with the isomorphism classes of indecomposable non-projective modules in $\operatorname{MCM}(A)$. 
Proof. Non-trivial direct summands of an object in an additive category correspond to non-trivial idempotents in its endomorphism ring.

Let $M$ be an indecomposable non-projective in $\operatorname{MCM}(A)$. Then $E=\operatorname{End}_{\mathrm{Gr} A} M$ is a finite dimensional local $\operatorname{ring}$, meaning that $E / \operatorname{rad} E$ is a division ring. Hence the endomorphism ring of $M$ in $\underline{\operatorname{MCM}}(A)$ is also local, so $M$ is indecomposable in $\underline{\operatorname{MCM}}(A)$.

Let $M^{\prime}$ be another indecomposable non-projective in $\operatorname{MCM}(A)$, and suppose that $f: M \rightarrow M^{\prime}$ and $g: M^{\prime} \rightarrow M$ become mutually inverse isomorphisms in $\operatorname{MCM}(A)$. To show that $M$ is isomorphic to $M^{\prime}$ in $\operatorname{MCM}(A)$, it suffices to show that $f g$ and $g f$ are isomorphisms in $\operatorname{MCM}(A)$. It therefore suffices to show that if $h: M \rightarrow M$ is an isomorphism in $\underline{\operatorname{MCM}}(A)$, then it is an isomorphism in $\operatorname{MCM}(A)$. But this is clear, since the isomorphisms $M \rightarrow M$ in either category are the endomorphisms that are not in the radical.

We have shown that the functor $\operatorname{MCM}(A) \rightarrow \underline{\operatorname{MCM}}(A)$ gives an injective map from the set of isomorphism classes of indecomposable non-projective CohenMacaulay modules to the set of isomorphism classes of indecomposable objects in $\underline{\operatorname{MCM}}(A)$. We now show this map is surjective. If $M$ is a maximal Cohen-Macaulay module that becomes indecomposable as an object in $\underline{\operatorname{MCM}}(A)$ we may write $M$ as a direct sum of indecomposables in $\operatorname{MCM}(A)$ and this gives a direct sum decomposition of $M$ in $\operatorname{MCM}(A)$ each term of which is either zero or indecomposable; hence, in $\operatorname{MCM}(A), M$ is isomorphic to some $M^{\prime}$ where $M^{\prime}$ is an indecomposable non-projective in $\operatorname{MCM}(A)$.

Remark. Suppose that $A$ is Gorenstein, and let $N$ be a maximal Cohen-Macaulay module having no non-zero projective direct summand. By applying $\underline{\operatorname{Hom}}_{A}(-, A)$ to $0 \rightarrow \Omega N \rightarrow P \rightarrow N \rightarrow 0$, where $P \rightarrow N \rightarrow 0$ is the start of a minimal projective resolution, one sees that $\Omega N$ also has no non-zero projective direct summand. Hence for all $d \geq 0, \Omega^{d} N$ has no non-zero projective direct summands.

\subsection{Simple objects and maximal Cohen-Macaulay modules.}

Lemma 3.5. Let A be a connected, graded, Gorenstein, Koszul algebra and

$$
B: \underline{\operatorname{MCM}}(A) \rightarrow \mathrm{D}^{\mathrm{b}}\left(\operatorname{qgr} A^{!}\right)
$$

the equivalence in Theorem 3.2. Let $S$ be a simple object in $\mathrm{qgr} A^{!}$. Then

(1) there is a unique-up-to-isomorphism indecomposable $M \in \operatorname{MCM}(A)$ such that $B M \cong S[0] ;$

(2) if $S \cong S(d)$, then $\Omega^{d} M(d) \cong M$;

(3) $\Omega^{n} M(n)$ has a linear resolution for all $n$.

Proof. The equivalence of categories and Lemma 3.4 ensure the existence and uniqueness of $M$. Because $S[0]$ is in the heart of $\mathrm{D}^{\mathrm{b}}\left(\mathrm{qgr} A^{!}\right), M$ has a linear resolution. 
An induction argument using Lemma 3.3 shows that $B\left(\Omega^{d} M(d)\right) \cong(B M)(d) \cong$ $S(d) \cong S$, so $M \cong \Omega^{d} M(d)$ in $\underline{\operatorname{MCM}}(A)$. Because $M$ is indecomposable, it follows that $M$ is isomorphic to a direct summand of $\Omega^{d} M(d)$ in $\operatorname{MCM}(A)$ and the complementary summand is projective. By the previous remark, $\Omega^{d} M$ has no non-zero projective direct summand, so $\Omega^{d} M(d) \cong M$.

Since $M$ has a linear resolution, so does each $\Omega^{n} M(n)$.

\section{Smoothness}

A noncommutative space $X$ is smooth of global dimension $d$ if $d$ is the largest integer such that $\operatorname{Ext}_{X}^{d}(M, N) \neq 0$ for some $X$-modules $M$ and $N$.

We now consider the question of what homological properties of a connected graded $k$-algebra $A$ imply that $\operatorname{Proj} A$ is smooth.

4.1. The following summarizes the commutative case.

Proposition 4.1. Let $A$ be a graded quotient of a positively graded polynomial ring. Write $X=\operatorname{Proj} A$. The following are equivalent:

(1) $\operatorname{gldim} X \leq d$;

(2) $\operatorname{dim}_{k} \operatorname{Ext}_{A}^{i}(M, N)<\infty$ for all $i \geq d+1$ and all $M, N \in \operatorname{gr} A$;

(3) whenever $N \rightarrow E^{\bullet}$ is a minimal injective resolution in $\mathrm{Gr} A, E^{i}$ is torsion for all $i \geq d+1$.

Proof. (3) $\Longleftrightarrow$ (1) If $0 \rightarrow N \rightarrow E^{\bullet}$ is a minimal injective resolution in $\operatorname{Gr} A$, then $0 \rightarrow \pi N \rightarrow \pi E^{\bullet}$ is a minimal injective resolution in Qcoh $X$. Thus gldim $X \leq d$ if and only if $\pi E^{i}=0$ for all $i \geq d+1$. Hence the equivalence of conditions (1) and (3)

(2) $\Longrightarrow$ (1) Since $A$ is commutative, $\operatorname{Ext}_{A}^{i}(M, N)$ is an $A$-module. If $f$ is a homogeneous element of $A$ lying in $\mathfrak{m}$, then $A\left[f^{-1}\right] \otimes_{A} \underline{\operatorname{Ext}}_{A}^{i}(M, N)=0$ for $i>d$, and so $\underline{\operatorname{Ext}}_{A\left[f^{-1}\right]}^{i} \equiv 0$ for $i>d$. Hence Proj $A\left[f^{-1}\right]$ is smooth of global dimension at most $d$. Since $X$ is covered by open affines of the form Spec $A\left[f^{-1}\right]_{0} \cong \operatorname{Proj} A\left[f^{-1}\right]$ with $f \in \mathfrak{m}$, it follows that gldim $X \leq d$.

(3) $\Longrightarrow$ (2) If condition (3) holds then applying $\underline{\operatorname{Hom}}_{A}(M,-)$ to a minimal injective resolution of $N$ produces a complex consisting of torsion modules after the $d^{\text {th }}$ term. Hence $\operatorname{Ext}_{A}^{i}(M, N)$ is torsion for $i>d$. However, if $M$ and $N$ are noetherian, then $\underline{\operatorname{Ext}}_{A}^{i}(M, N)$ is a noetherian $A$-module as one sees by applying $\underline{\operatorname{Hom}}_{A}(-, N)$ to a minimal projective resolution of $M$. Hence $\underline{\operatorname{Ext}}_{A}^{i}(M, N)$ is finite dimensional for $i>d$ whenever $M, N \in \operatorname{gr} A$.

The proof of (3) $\Longleftrightarrow$ (1) works when $A$ is not commutative, but the other two parts of the proof fail because $\operatorname{Ext}_{A}^{i}(M, N)$ is not an $A$-module when $A$ is not commutative. Nevertheless, we will show that the implication (3) $\Longrightarrow(2)$ holds if $A$ satisfies $\chi$. 
First we need the following lemma that we learned from Kontsevich.

Lemma 4.2. Let $A$ be a noetherian connected graded $k$-algebra satisfying $\chi$. If $\operatorname{gldim}(\operatorname{Proj} A)=d<\infty$ and $M \in \operatorname{gr} A$, then there is a perfect complex $P \in$ $\mathrm{D}^{\mathrm{b}}(\operatorname{gr} A)$ concentrated in homological degree $[-d, 0]$ and a bounded complex $N$ together with a map in $M_{\geq n} \oplus N \rightarrow P$ (n large) whose cone has finite dimensional cohomology.

Proof. Take an exact sequence $0 \rightarrow Z \rightarrow P_{d} \rightarrow \cdots \rightarrow P_{0} \rightarrow M \rightarrow 0$ with each $P_{i}$ a finitely generated free module. Applying $\pi$ to this gives an element of $\operatorname{Ext}_{X}^{d+1}(\pi M, \pi Z)$ which must be zero, so the triangle $\pi Z[d] \rightarrow \pi P \rightarrow \pi M \rightarrow$ is split. Applying $\omega$ gives an isomorphism $\omega \pi M \oplus \omega \pi Z[d] \rightarrow \omega \pi P$. However, since $\chi$ holds, for every finitely generated module $N$ the map $N_{\geq n} \rightarrow(\omega \pi N)_{\geq n}$ has finite dimensional cokernel (and it obviously has finite dimensional kernel). Hence, for large $n$ there is an isomorphism $M_{\geq n} \oplus Z[d]_{\geq n} \cong P_{\geq n}$, and hence a map $M_{\geq n} \oplus Z[d]_{\geq n} \rightarrow P$ whose cone has finite dimensional cohomology.

Proposition 4.3. Let $A$ be a noetherian connected graded $k$-algebra satisfying $\chi$. Write $X=\operatorname{Proj} A$. If gldim $X=d<\infty$, then $\operatorname{dim}_{k} \underline{\operatorname{Ext}}_{A}^{i}(M, N)<\infty$ for all $i \geq d+1$ and all $M, N \in \operatorname{gr} A$.

Proof. By Lemma 4.2, there is a distinguished triangle $M_{\geq n} \oplus N \rightarrow P \rightarrow C \rightarrow$ such that $\bigoplus_{q} H^{q}(C)$ is finite dimensional.

4.2. We call a triangulated category is semisimple if in every distinguished triangle

$$
L \stackrel{u}{\rightarrow} M \stackrel{v}{\rightarrow} N \stackrel{w}{\rightarrow}
$$

at least one of $u, v$, and $w$ is zero. The condition that $w=0$ is equivalent to the condition that $u$ and $v$ induce an isomorphism $M \cong L \oplus N$. It follows that if $L$ and $M$ are objects in a semisimple triangulated category, then $\operatorname{Hom}(L, M) \neq 0$ if and only if $\operatorname{Hom}(M, L) \neq 0$. Furthermore, the heart of every $t$-structure on a semisimple triangulated category is semisimple, meaning that every short exact sequence splits. The derived category of a semisimple abelian category is semisimple.

Notation. We write $\mathrm{MCM}_{\geq n}$ for the full subcategory of $\operatorname{MCM}(A)$ consisting of graded Cohen-Macaulay $A$-modules $M$ such that $M_{i}=0$ for all $i<n$. We write $\underline{\mathrm{MCM}}_{\geq n}$ for the essential image of $\mathrm{MCM}_{\geq n}$ in $\underline{\operatorname{MCM}}(A)$.

Proposition 4.4. Let $A$ be a connected, graded $k$-algebra that is Gorenstein and satisfies $\chi$. If $\underline{\operatorname{MCM}}(A)$ is semisimple, then $\operatorname{Proj} A$ is smooth.

Proof. Suppose that $A$ has depth $d$, so the $d^{\text {th }}$ syzygy of a finitely generated module is maximal Cohen-Macaulay. 
Fix $K \in \operatorname{MCM}(A)$. For all integers $n$ greater than the degrees of the minimal generators of $K, \operatorname{Hom}_{\underline{\mathrm{MCM}}}(K,-)$ vanishes on $\underline{\operatorname{MCM}}_{\geq n}$. Since $\underline{\operatorname{MCM}}(A)$ is semisimple, it follows that $\operatorname{Hom}_{\underline{\mathrm{MCM}}}(-, K)$ also vanishes on $\underline{\mathrm{MCM}}_{\geq n}$.

Now fix $M, N \in \operatorname{gr} A$. Then

$$
\begin{aligned}
\operatorname{Ext}_{\operatorname{Proj} A}^{d+1}(\pi M, \pi N) & \cong \operatorname{Ext}_{\mathrm{Gr} A}^{d+1}\left(M_{\geq n}, N\right) \quad \text { for } n \gg 0 \\
& \cong \operatorname{Ext}_{\mathrm{Gr} A}^{1}\left(\Omega^{d}\left(M_{\geq n}\right), N\right) \\
& \cong \operatorname{Ext}_{\mathrm{Gr} A}^{d+1}\left(\Omega^{d}\left(M_{\geq n}\right), \Omega^{d} N\right) \quad \text { (because } A \text { is Gorenstein) } \\
& \cong \operatorname{Hom}_{\underline{\mathrm{MCM}}}\left(\Omega^{d}\left(M_{\geq n}\right),\left(\Omega^{d} N\right)[d+1]\right) .
\end{aligned}
$$

The previous paragraph shows that this is zero for $n \gg 0$ because $\Omega^{d}\left(M_{\geq n}\right) \in$ $\mathrm{MCM}_{\geq n}$.

Part of the argument in Proposition 4.4 can be restated in the following way.

Proposition 4.5. Suppose that $\underline{\operatorname{MCM}}(A)$ is semisimple. If $N \in \operatorname{MCM}(A)$, there is an integer $n$ such that $\operatorname{Ext}_{\mathrm{Gr} A}^{1}(M, N)=0$ for all $M \in \mathrm{MCM}_{\geq n}$.

Proof. Choose an integer $n>$ \{the degrees of a minimal set of generators for $N$ \}. Suppose that $M \in \mathrm{MCM}_{\geq n}$. Then $\Omega M \in \mathrm{MCM}_{\geq n}$ also. Hence $\operatorname{Hom}_{\mathrm{Gr} A}(N, \Omega M)$ is zero, and so is its quotient $\operatorname{Hom}_{\mathrm{MCM}(A)}(N, M[-1])$. $\operatorname{But} \underline{\operatorname{MCM}}(A)$ is semisimple, so $\operatorname{Hom}_{\underline{\mathrm{MCM}}(A)}(M[-1], N)=0$ also. Thus $\operatorname{Ext}_{\mathrm{Gr} A}^{1}(M, N)=0$.

Proposition 4.6. Let $S$ be a Gorenstein k-algebra of finite global dimension and $z$ a central regular element of degree $d$. Let $A=S /(z)$. If $M \in \operatorname{MCM}(A)$ is not projective, then

(1) there is a resolution $0 \rightarrow S^{s} \rightarrow S^{s} \rightarrow M \rightarrow 0$ of ungraded $S$-modules;

(2) $\Omega^{2} M \cong M(-d)$

(3) $\operatorname{Ext}_{A}^{i+2}(M, N) \cong \underline{\operatorname{Ext}}_{A}^{i}(M, N)(d)$ for all $A$-modules $N$ and all $i \geq 1$.

Proof. (1) We have depth $M=\operatorname{depth}_{A} M=\operatorname{depth}_{A} A=\operatorname{depth}_{S} A=\operatorname{depth} S-1$, so $\operatorname{pdim}_{S} M=1$ by the Auslander-Buchsbaum formula. Hence $M$ has a free resolution $0 \rightarrow S^{r} \rightarrow S^{s} \rightarrow M \rightarrow 0$. But $r \leq s$ because $S$ is noetherian and $s \leq r$ because $S^{s} z \subset S^{r}$, so $s=r$.

Although this is a resolution of $M$ in QGr $S$, the minimal resolution of $M$ in $\operatorname{Gr} S$ also has this form although we may have to change $s$ and we will need to place some gradings on the two free modules.

(2) and (3): From the presentation of $A$ we get $\operatorname{Tor}_{1}^{S}(M, A) \cong M(-d)$. Now, by applying $-\otimes_{S} A$ to the resolution of $M_{S}$, we obtain an exact sequence

$$
0 \rightarrow M(-d) \rightarrow A^{s} \rightarrow A^{s} \rightarrow M \rightarrow 0
$$


in $\operatorname{Mod} A$. We can give the two copies of $A^{s}$ gradings such that this becomes a sequence of graded $A$-modules. Thus $\Omega^{2} M \cong M(-d)$. The result now follows by dimension-shifting.

\section{Quadrics in quantum $\mathbb{P}^{3} \mathbf{s}$}

5.1. The algebra $C(\boldsymbol{A})$. Using the notation in part (2) of the next lemma, we define

$$
C(A):=A^{!}\left[w^{-1}\right]_{0} .
$$

We write Mod $C$ for the category of right modules over a ring $C$, and $\bmod C$ for the full subcategory of finitely presented modules.

Lemma 5.1. Let $S$ be a connected, graded, noetherian, Koszul algebra of finite global dimension, $z$ a central regular element of degree two, and $A=S /(z)$. Then

(1) A is a Koszul algebra;

(2) there is a central, regular element $w \in A_{2}^{!}$such that $A^{!} /(w)=S^{!}$;

(3) the algebra $C(A)$ has finite dimension equal to $\operatorname{dim}_{k}\left(S^{!}\right)^{(2)}$, the dimension of the even degree part of $S$;

(4) the categories $\mathrm{QGr} A^{!}$and $\operatorname{Mod} C(A)$ are equivalent via $\pi N \mapsto N\left[w^{-1}\right]_{0}$, where $N \in \operatorname{Gr} A^{!}$.

Proof. (1) and (2): The proof is similar to that for modding out a central regular element of degree one [10].

(3) Because $S$ is Koszul, the hypothesis that $\operatorname{gldim} S<\infty$ implies that $S^{\text {! }}$ has finite dimension. It follows that $A_{m+2}^{!}=w A_{m}^{!}$for large $m$, and hence that

$$
A^{!}\left[w^{-1}\right]_{0}=A_{0}^{!}+A_{2}^{!} w^{-1}+\cdots=A_{2 n}^{!} w^{-n}
$$

for $n \gg 0$. In particular, $\operatorname{dim}_{k} A\left[w^{-1}\right]_{0}=\operatorname{dim}_{k} A_{2 n}$ for $n \gg 0$. By (1) and (2), $(1+t) H_{A^{!}}(t)=(1-t)^{-1} H_{S^{!}}(t)$, so

$$
\operatorname{dim}_{k} A_{2 n}^{!}=\operatorname{dim}_{k} S_{0}^{!}+\operatorname{dim}_{k} S_{2}^{!}+\cdots,
$$

for $n \gg 0$, as required.

(4) A graded $A^{!}$-module has finite dimension if and only if it is annihilated by a power of $w$, so $\operatorname{Gr} A^{!} / \operatorname{Fdim} A^{!}$is equivalent to $\operatorname{Gr} A^{!}\left[w^{-1}\right]$. Since $A^{!}$is generated in degree one, $A^{!}\left[w^{-1}\right]$ is strongly graded, and therefore $\operatorname{Gr} A^{!}\left[w^{-1}\right]$ is equivalent to $\operatorname{Mod} A^{!}\left[w^{-1}\right]_{0}$.

The degree shift functor (1) on $\operatorname{Gr} A^{!}$induces auto-equivalences of $\mathrm{QGr} A^{!}$and Mod $C(A)$ that we still denote by (1). Since $w$ is central and homogeneous of degree two, on $\operatorname{Mod} C(A)$ we have $(2) \cong \operatorname{id}_{\operatorname{Mod} C(A)}$.

Notice that $A^{!}$is noetherian because $A^{!} /(w)$ is. 
Proposition 5.2. Let $S$ be a Gorenstein, connected, graded, noetherian, Koszul algebra of finite global dimension, $z$ a central regular element of degree two, and $A=S /(z)$.

(1) There are equivalences of categories

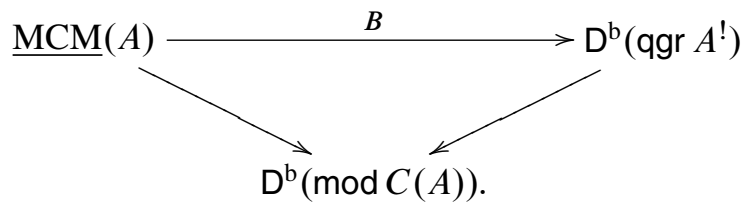

(2) If $C(A)$ is a semisimple ring, then Proj $A$ is smooth.

Proof. The horizontal equivalence is given by Theorem 3.2, and the southwest equivalence is given by Lemma 5.1. Part (2) follows from (1) and Proposition 4.4 because the derived category of a semisimple abelian category is semsimple, hence abelian.

5.2. Notation and Hypotheses. We fix the following hypotheses and notation for the remainder of this section: $k$ is an algebraically closed field, $S$ denotes a connected, graded, noetherian, Gorenstein, Koszul algebra with Hilbert series $(1-t)^{-4} ; z$ is a non-zero, homogeneous, central element of degree two such that $A:=S /(z)$ is a domain. We write $Q:=\operatorname{Proj} A$.

The hypotheses imply that $H_{S}(t)=(1+t)^{4}$, so gldim $S=4$. The previous two results apply, so the finite dimensional algebra $C(A)$ is well-defined.

It follows from Corollary 6.7 below and [1], Thm. 3.9, that $S$ is a domain. Thus Proj $S$ is a quantum $\mathbb{P}^{3}$ and $Q$ is a quadric hypersurface in it. The assumption that $A$ is a domain says that $Q$ is "reduced and irreducible".

Proposition 5.3. Suppose that $S$ is a connected, graded, noetherian, Gorenstein, Koszul algebra such that $H_{S}(t)=(1-t)^{-4}$. Let $0 \neq z \in S_{2}$ be a central element and suppose that $A:=S /(z)$ is a domain. Then

(1) $\operatorname{dim}_{k} C(A)=8$;

(2) $C(A)$ has no 1-dimensional modules;

(3) the following are equivalent:

(a) $C(A)$ is semisimple;

(b) $C(A)$ has two simple modules up to isomorphism;

(c) $C(A) \cong M_{2}(k) \oplus M_{2}(k)$.

Proof. (1) This does not depend on $A$ being a domain. Because $z$ is regular, $H_{A}(t)=$ $(1+t)(1-t)^{-3}$ and $H_{A^{!}}(t)=(1-t)^{-1}(1+t)^{3}$. Thus $\operatorname{dim}_{k} A_{n}=8$ for $n \gg 0$, and $\operatorname{dim}_{k} C(A)=8$ by Lemma 5.1 . 
(2) First we show that if $V$ is a subspace of $A_{1}^{!}=A_{1}^{*}$ of codimension one, then $A_{1}^{!} V=V A_{1}^{!}=A_{2}^{!}$. To see this write $V=a^{\perp}$ where $a \in A_{1}$; because $A$ is a domain, $a \otimes A_{1} \cap R=0$, where $R$ denotes the relations in $A_{1} \otimes A_{1}$ defining $A$; hence $a^{\perp} \otimes A_{1}^{*}+R^{\perp}=A_{1}^{*} \otimes A_{1}^{*}$; thus $V A_{1}^{!}=A_{2}^{!}$. Similarly, $A_{1}^{!} V=A_{2}^{!}$.

Claim: Let $T$ be a connected graded algebra generated in degree one, and $w \in T_{d}$ a central regular element of degree $d>1$. If $T_{2}=T_{1} V$ for every codimension one subspace $V \subset T_{1}$, then $T\left[w^{-1}\right]_{0}$ does not have a 1-dimensional module.

Proof. Suppose to the contrary that $N_{0}$ is a 1-dimensional $T\left[w^{-1}\right]_{0}$-module. Then $N_{0}$ is the degree zero component of the $T\left[w^{-1}\right]$-module $N:=T\left[w^{-1}\right] \otimes_{T\left[w^{-1}\right]_{0}} N_{0}$. Because $w$ is a unit, $N_{d i}=w^{i} N_{0}$ for all $i \in \mathbb{Z}$. In particular, $\operatorname{dim}_{k} N_{d i}=1$ for all $i$. Hence if $m \in N_{d i-1}$, then $V m=0$ for some subspace $V \subset T_{1}$ of codimension at most one. Hence $T_{2} m=0$. It follows that

$$
0=T_{2} N_{d i-1}=T_{3} N_{d i-2}=\cdots=T_{d+1} N_{d i-d} .
$$

In particular, $T_{d+1} N=0$, so $w^{2} N=0$, contradicting the fact that $w$ is a unit in $T\left[w^{-1}\right]$.

The claim applies to $T=A^{!}$, so (2) follows.

(3) This follows from (1) and (2) because $k$ is algebraically closed.

5.3. The set $M$. We define

$$
\mathrm{M}:=\left\{M \in \operatorname{MCM}(A) \mid M \text { is indecomposable, } M_{0} \cong k^{2}, M=M_{0} A\right\} .
$$

Because $A$ is a noetherian domain it has a division ring of fractions, say $Q$, and we may define the rank of an $A$-module $N$ by $\operatorname{dim}_{Q} N \otimes_{A} Q$.

Proposition 5.4. Let $A$ and $S$ be as in Proposition 5.3. If $M \in \mathbb{M}$, then

(1) $M \cong \Omega^{2} M(2)$;

(2) its minimal resolution is $\cdots \rightarrow A(-2)^{2} \rightarrow A(-1)^{2} \rightarrow A^{2} \rightarrow M \rightarrow 0$;

(3) $\operatorname{rank} M=1$;

(4) $H_{M}(t)=2(1-t)^{-3}$;

(5) $M$ is 3-critical with respect to GK-dimension.

Furthermore, there is a bijection

$$
\mathrm{M} \longleftrightarrow\{\text { simple } C(A) \text {-modules }\}, \quad M \longleftrightarrow F M .
$$

Proof. The hypotheses on $M$ ensure that it is not projective.

(1) This was already established in Proposition 4.6.

(2) By (1), the minimal resolution of $\Omega^{2} M$ begins $A(-2)^{2} \rightarrow \Omega^{2} M \rightarrow 0$.

Combining this with Proposition 4.6, we see that the minimal resolution of $M$ begins

$$
\cdots \rightarrow A(-2)^{2} \rightarrow A(-i) \oplus A(-j) \rightarrow A^{2} \rightarrow M \rightarrow 0
$$


for some $i, j$. However, the minimality of the resolution forces $i=j=1$. Since $\Omega^{2} M \cong M(-2)$, the full minimal resolution of $M$ can be constructed by splicing together shifts of the exact sequence $0 \rightarrow M(-2) \rightarrow A(-1)^{2} \rightarrow A^{2} \rightarrow M \rightarrow 0$.

(3) Because $A$ is a domain, its rank is one. The result now follows from the exact sequence $0 \rightarrow M(-2) \rightarrow A(-1)^{2} \rightarrow A^{2} \rightarrow M \rightarrow 0$.

(4) This follows from (2).

(5) Because $M(-1)$ embeds in $A^{2}$, every non-zero submodule of $M$ has GKdimension three; since the rank of $M$ is one, all its non-zero submodules have rank one too. Hence every proper quotient of $M$ has GK-dimension $\leq 2$.

We now establish the bijection between $\mathrm{M}$ and the simple $C(A)$-modules. Let $M \in \mathbb{M}$. By (2), $M$ has a linear resolution, so $F M=N[0]$ for some $C$-module $N$. But $N=\underline{\operatorname{Ext}}_{A}^{*}(M, k)\left[w^{-1}\right]_{0} \cong \operatorname{Ext}_{A}^{2 i}(M, k)$ for $i \gg 0$, so $\operatorname{dim}_{k} N=2$, and Proposition 5.3 now implies that $N$ is simple.

Conversely, let $N$ be a simple $C$-module. By Lemma 3.5, there is a unique indecomposable maximal Cohen-Macaulay module $M$ such that $F M \cong N[0]$, and $M$ has a linear resolution. By Proposition 5.3, $\operatorname{dim}_{k} N=2$. But $N \cong \operatorname{Ext}_{A}^{*}(M, k)\left[w^{-1}\right]_{0}$, so $\operatorname{Ext}_{A}^{2 i}(M, k) \cong k^{2}$ for $i \gg 0$. Hence $\Omega^{2 i} M$ is generated by two elements for $i \gg 0$. But $\Omega^{2 i} M \cong M(-2 i)$ by Proposition 4.6, so $M$ is generated by two elements, and these are of degree zero because $M$ has a linear resolution. Hence $M \in \mathbb{M}$.

Lemma 5.5. Let $A$ and $S$ be as in Proposition 5.3. If $M \in \mathbb{M}$, then

(1) $\Omega M(1) \in \mathbb{M}$;

(2) there is an exact sequence $0 \rightarrow M(-1) \rightarrow A^{2} \rightarrow \Omega M(1) \rightarrow 0$;

(3) if $C(A)$ is not semisimple, then $M \cong \Omega M(1)$;

(4) if $M \nsubseteq \Omega M(1)$, then $\operatorname{Hom}_{\mathrm{Gr} A}(M, \Omega M(1))=0$.

Proof. (1) By the remark after Lemma 3.4, $\Omega M(1)$ is indecomposable. From the minimal resolution for $M$ we see that $\Omega M(1)$ is generated in degree zero and that $\operatorname{dim}_{k} \Omega M(1)_{0}=2$.

(2) This follows from the fact that $\Omega^{2} M \cong M(-2)$.

(3) If $C(A)$ is not semisimple it has only one simple module, so, by (1) and the bijection in Proposition 5.4, $M \cong \Omega M(1)$.

(4) A non-zero degree-zero homomorphism $\alpha: M \rightarrow \Omega M$ (1) would be injective because $M$ and $\Omega M(1)$ are 3-critical with respect to GK-dimension, so its restriction $M_{0} \rightarrow(\Omega M(1))_{0}$ would be an isomorphism, whence $\alpha$ would be surjective. This would contradict the hypothesis that $M \nsubseteq \Omega M(1)$.

\subsection{Smoothness of $Q$ and semisimplicity of $C(A)$}

Theorem 5.6. The noncommutative quadric $Q$ is smooth if and only if $C(A)$ is semisimple. 
Proof. $\Longleftarrow:$ This was proved in Proposition 5.2.

$\Longrightarrow$ : We shall prove the contrapositive, so suppose that $C(A)$ is not semisimple. Then it has only one simple module, $N$ say. Let $M(1) \in \mathbb{M}$ be such that $F(M(1)) \cong$ $N[0]$. Thus $M$ is generated in degree one.

We write $\mathcal{M}$ for $\pi M$.

By Lemma $5.5, M \cong \Omega M(1)$, so there is an exact sequence

$$
0 \rightarrow M \rightarrow A^{2} \rightarrow M(1) \rightarrow 0 .
$$

This gives an exact sequence

$$
0 \rightarrow \mathcal{M} \rightarrow \mathcal{O}_{Q}^{2} \rightarrow \mathcal{M}(1) \rightarrow 0
$$

in $\operatorname{Mod} Q$.

The result that gldim $Q=\infty$ will be established in step 3 below.

Step 1 . $\operatorname{Ext}_{Q}^{1}(\mathcal{M}, \mathcal{M}) \neq 0$.

Proof. Because $M$ is maximal Cohen-Macaulay

$$
\operatorname{Ext}_{\mathrm{Gr} A}^{1}(M, M) \cong \operatorname{Hom}_{\underline{\mathrm{MCM}}(A)}(M, M[1]) .
$$

Applying the contravariant equivalence $F$ this is isomorphic to

$$
\operatorname{Hom}_{\mathrm{D}(C(A))}(F(M[1]), F M) \cong \operatorname{Hom}_{\mathrm{D}(C(A))}(F M,(F M)[1]) .
$$

But $F M$ is a translate of the unique simple $C$-module $N$, so the last term is isomorphic to $\operatorname{Ext}_{C(A)}^{1}(N, N)$ which is non-zero because $C(A)$ is not semisimple. Hence $\operatorname{Ext}_{\mathrm{Gr} A}^{1}(M, M) \neq 0$.

Applying $\pi$ to a non-split exact sequence

$$
0 \rightarrow M \rightarrow D \rightarrow M \rightarrow 0
$$

in $\mathrm{Gr} A$ gives an exact sequence

$$
0 \rightarrow \mathcal{M} \rightarrow \mathscr{D} \rightarrow \mathcal{M} \rightarrow 0
$$

in $\operatorname{Mod} Q$. Write $\theta: \operatorname{MCM}(A) \rightarrow \operatorname{Gr} A$ for the inclusion functor. There is an exact sequence of functors $0 \rightarrow H_{\mathfrak{m}}^{0} \rightarrow \operatorname{id}_{\operatorname{Gr} A} \rightarrow \omega \pi \rightarrow H_{\mathfrak{m}}^{1} \rightarrow 0$. These two local cohomology functors vanish on $\operatorname{MCM}(A)$, so there is an isomorphism of functors $\theta \rightarrow \omega \pi \theta$. Hence, if (5.3) were to split via a map $g: \mathcal{M} \rightarrow \mathscr{D}$, then $\omega(g)$ would provide a splitting of (5.2). It follows that $\operatorname{Ext}_{Q}^{1}(\mathcal{M}, \mathcal{M}) \neq 0$.

Step 2. $\operatorname{Ext}_{Q}^{2}(\mathcal{M}(1), \mathcal{M}) \neq 0$.

Proof. Applying $\operatorname{Hom}_{Q}(-, \mathcal{M})$ to (5.1) gives an exact sequence

$$
\operatorname{Ext}_{Q}^{1}\left(\mathcal{O}_{Q}, \mathcal{M}\right)^{2} \rightarrow \operatorname{Ext}_{Q}^{1}(\mathcal{M}, \mathcal{M}) \rightarrow \operatorname{Ext}_{Q}^{2}(\mathcal{M}(1), \mathcal{M}) .
$$


The first term is zero because depth $M=3$ implies that

$$
0=H_{\mathfrak{m}}^{2}(M) \cong H^{1}(Q, \mathcal{M})=\operatorname{Ext}_{Q}^{1}\left(\mathcal{O}_{Q}, \mathcal{M}\right) .
$$

The second term is non-zero by step 1 , so the third term is also non-zero, as required.

Step 3. $\operatorname{Ext}_{Q}^{n}(\mathcal{M}(n-1), \mathcal{M}) \neq 0$ for all $n \geq 2$.

Proof. We argue by induction on $n$. The case $n=2$ has already been established in Step 2. Applying $\operatorname{Hom}_{Q}(\mathcal{M}(n),-)$ to (5.1) gives an exact sequence

$$
\operatorname{Ext}_{Q}^{n}\left(\mathcal{M}(n), \mathcal{O}_{Q}\right)^{2} \rightarrow \operatorname{Ext}_{Q}^{n}(\mathcal{M}(n), \mathcal{M}(1)) \rightarrow \operatorname{Ext}_{Q}^{n+1}(\mathcal{M}(n), \mathcal{M})
$$

The first term is isomorphic to two copies of $\operatorname{Ext}_{Q}^{n}\left(\mathcal{M}(n-2), \mathcal{O}_{Q}(-2)\right)$ which is isomorphic to $H^{2-n}(Q, \mathcal{M}(n-2))^{*}$ by Serre duality. This is zero for $n \geq 3$, and if $n=2$ it is isomorphic to $\operatorname{Hom}_{\mathrm{Gr} A}(A, M)^{*}=M_{0}$ which is zero because $M$ is generated in degree one. Since the first term of (5.4) is zero for all $n \geq 2$, we see from the other two terms that the induction argument goes through.

Corollary 5.7. $C(A)$ is semisimple if and only if $M \not \Omega M(1)$ for all $M \in M$.

Proof. $\Longleftarrow:$ If $C$ were not semisimple it would have a unique simple module so, up to isomorphism, there would be only one module in $\mathrm{M}$; but if $M$ is in $\mathrm{M}$ so is $\Omega M(1)$, whence $M \cong \Omega M(1)$.

$\Longrightarrow$ : If $C$ is semisimple, then gldim $Q<\infty$. But the proof of Theorem 5.6 showed that if there were an $M$ in $M$ such that $M \cong \Omega M(1)$, then $\operatorname{gldim} Q=\infty$. Hence there can be no such $M$.

Corollary 5.8. If $Q$ is smooth, then $\mathrm{M}$ consists of two non-isomorphic modules, say $\mathrm{M}=\left\{M, M^{\prime}\right\}$, and there are exact sequences

$$
0 \rightarrow M(-1) \rightarrow A^{2} \rightarrow M^{\prime} \rightarrow 0
$$

and

$$
0 \rightarrow M^{\prime}(-1) \rightarrow A^{2} \rightarrow M \rightarrow 0 .
$$

Proof. This follows immediately from Lemma 5.5 and Corollary 5.7.

\section{The Auslander property}

We fix the following notation in this section: $S$ denotes a connected, graded, Gorenstein, Koszul algebra with Hilbert series $(1-t)^{-4} ; z$ is a non-zero, homogeneous, central element of degree two and $A:=S /(z)$.

The main result in this section, Theorem 6.6, shows that $A$ has the Auslander property by which we mean that if $M \in \operatorname{gr} A$ and $N$ is a graded submodule of $\operatorname{Ext}_{A}^{j}(M, A)$ for some $j$, then $\operatorname{Ext}_{A}^{i}(N, A)=0$ for $i<j$. By [11], this will imply that $S$ also has the Auslander property. 
6.1. The grade of $M \in \operatorname{gr} A$ is $j(M):=\inf \left\{j \mid \underline{\operatorname{Ext}}_{A}^{j}(M, A) \neq 0\right\}$. The Auslander property is equivalent to the condition that $j(N) \geq j$ for all submodules $N \subset$ $\underline{\operatorname{Ext}}_{A}^{j}(M, A)$. To prove that $A$ has the Auslander property we first prove that

$$
j(M)+\operatorname{GKdim} M=3
$$

for all $M \in \operatorname{gr} A$.

The arguments in this section are close to those in [1], Sect. 4.

The following result is standard.

Lemma 6.1. If $R$ is a prime noetherian $k$-algebra of finite $G K$-dimension and $N \in$ $\bmod R$, the following are equivalent:

(1) $\operatorname{GKdim} N=\operatorname{GKdim} R$;

(2) $j(N)=0$;

(3) $N \otimes_{R} Q \neq 0$, where $Q=$ Fract $R$.

Because $A$ is Gorenstein its dualizing module $\omega_{A}$ is invertible, hence isomorphic to $A(\ell)$ for some integer $\ell$ as a left and as a right module. Our arguments in this section involve an examination of the convergent spectral sequence

$$
E_{2}^{p, q}=\underline{\operatorname{Ext}}_{A}^{p}\left(\underline{\operatorname{Ext}}_{A}^{-q}\left(M, \omega_{A}\right), \omega_{A}\right) \Rightarrow \mathbb{Q}^{p+q}(M)= \begin{cases}M & \text { if } p+q=0, \\ 0 & \text { if } p+q \neq 0 .\end{cases}
$$

We will often omit the subscript from $E_{2}^{p q}$.

Theorem 6.2. Let $A$ be as above and let $M \in \operatorname{gr} A$. The $E_{2}$-page of the spectral sequence (6.1) looks like

$$
\begin{array}{cccc}
E^{00} & E^{10} & 0 & 0 \\
0 & E^{1,-1} & E^{2,-1} & E^{3,-1} \\
0 & 0 & E^{2,-2} & E^{3,-2} \\
0 & 0 & 0 & E^{3,-3}
\end{array}
$$

Proof. Since $\operatorname{Ext}_{A}^{i}(-, A)=0$ for $i>3$, the non-zero terms on the $E_{2}$-page of the double-Ext spectral sequence lie in the $(4 \times 4)$-region depicted. Therefore the $E^{20}$ and $E^{30}$ terms survive to the $E_{\infty}$-page. But any non-zero terms on the $E_{\infty}$-page must lie on the diagonal, so $E^{20}=E^{30}=0$. Now $\operatorname{Ext}_{A}^{3}\left(M, \omega_{A}\right) \cong H_{\mathfrak{m}}^{0}(M)^{*}$ is finite dimensional, so is Cohen-Macaulay of depth zero, whence $E^{p 3}=0$ for $p<3$. This explains the zeroes in the top and bottom rows of (6.2).

The division ring of fractions, $Q=$ Fract $A$, is flat as a left and as a right $A$-module and $\operatorname{gldim} Q=0$ so, for $i>0$,

$$
0=\operatorname{Ext}_{Q}^{i}\left(M \otimes_{A} Q, A \otimes_{A} Q\right) \cong Q \otimes_{A} \underline{\operatorname{Ext}}_{A}^{i}(M, A) .
$$


Applying Lemma 6.1 to $N=\underline{\operatorname{Ext}}_{A}^{i}(M, A)$, we obtain $E^{0,-1}=E^{0,-2}=E^{0,-3}=0$, giving the zeroes in the left-most column of (6.2).

It remains to show that $E^{1,-2}=0$. Set $L=\operatorname{Ext}_{A}^{2}(M, A)$; if $L=0$ there is nothing to do, so suppose that $L \neq 0$. Since $\operatorname{ker}\left(E^{1,-2} \rightarrow E^{3,-3}\right)$ survives to the $E_{\infty}$-page, it is zero. Since $E^{3,-3}$ is finite dimensional so is $E^{1,-2}=\operatorname{Ext}_{A}^{1}(L, A)<\infty$. If $\underline{\operatorname{Ext}}_{A}^{1}(L, A)=0$ we are finished, so suppose otherwise.

Consider the $E_{2}$-page of the spectral sequence for $L$. Since

$$
Q \otimes_{A} L \cong \underline{\operatorname{Ext}}_{Q}^{2}\left(M \otimes_{A} Q, A \otimes_{A} Q\right)=0,
$$

$\underline{\operatorname{Hom}}_{A}(L, A)$ is zero; hence the $q=0$ and $q=-1$ rows look like

$$
\begin{array}{cccc}
0 & 0 & 0 & 0 \\
E^{0,-1} & E^{1,-1} & E^{2,-1} & E^{3,-1}
\end{array}
$$

Since $\underline{\operatorname{Ext}}_{A}^{1}(L, A)$ is non-zero and finite dimensional, $\underline{\operatorname{Ext}}_{A}^{3}\left(\underline{\operatorname{Ext}}_{A}^{1}(L, A), A\right) \neq 0$. But the $E^{3,-1}$ term survives to the $E_{\infty}$-page, so must be zero. From this contradiction we conclude that $\operatorname{Ext}_{A}^{1}(L, A)=0$, as required.

Lemma 6.3. If $M \in \operatorname{gr} S$ is a Cohen-Macaulay module, then

$$
\operatorname{depth} M+\operatorname{GKdim} M \equiv 0(\bmod 2) .
$$

Proof. The lemma is true for any connected graded Gorenstein algebra $S$ of finite global dimension, $n$ say, having Hilbert series of the form $f(t)(1-t)^{-n}$ where $f(t) \in \mathbb{Z}[t]$. The functional equation [1], (2.35), relating the Hilbert series of a module to that of its dual becomes

$$
H_{M \vee}(t)=(-1)^{d} H_{M}\left(t^{-1}\right)
$$

when $M$ is a Cohen-Macaulay module of depth $d$ and $M^{\vee}=\operatorname{Ext}_{S}^{n-d}\left(M, \omega_{S}\right)$. If $\operatorname{GKdim} M=r$, then $H_{M}(t)=g(t)(1-t)^{-r}$ for some $g(t) \in \mathbb{Z}\left[t, t^{-1}\right]$, so $H_{M} \vee(t)=(-1)^{d+r} t^{r} g\left(t^{-1}\right)(1-t)^{-r}$. However,

$$
\lim _{t \uparrow 1} H_{M}(t)
$$

is positive if $M \neq 0$ and the same applies to $H_{M \vee}(t)$, so $d+r$ is even.

Lemma 6.4. Let $A$ be as above and $M \in \operatorname{gr} A$. Suppose that $j(M)=1$ and write $M^{\vee}:=\underline{\operatorname{Ext}}_{A}^{1}(M, A)$. Then $M^{\vee}$ is Cohen-Macaulay of depth two and GK-dimension two.

Proof. Since $j(M)=1$, the $E_{2}$-page of the spectral sequence for $M$ looks like

$\begin{array}{cccc}0 & 0 & 0 & 0 \\ 0 & E^{1,-1} & E^{2,-1} & E^{3,-1} \\ 0 & 0 & E^{2,-2} & E^{3,-2} \\ 0 & 0 & 0 & E^{3,-3}\end{array}$


Both $E^{2,-1}$ and $E^{3,-1}$ survive to the $E_{\infty}$-page, so must be zero. Hence $M^{\vee}$ is Cohen-Macaulay of depth two and, by Lemma 6.3, GKdim $M^{\vee}=2$.

Theorem 6.5. If $A$ is as above, then

$$
j(M)+\operatorname{GKdim} M=3
$$

for all non-zero finitely generated graded $A$-modules $M$.

Proof. By Lemma 6.1, (6.3) holds when $M=\operatorname{GKdim} 3$ and when $j(M)=0$. Since finite dimensional modules are precisely the Cohen-Macaulay modules of depth zero, (6.3) holds when GKdim $M=0$ too, so it remains to prove (6.3) for modules of GKdimensions 1 and 2 .

Suppose that GKdim $M=1$. By Lemma 6.1, $j(M) \geq 1$. As in the proof of Lemma 6.4 , the $E_{2}$-page of the double-Ext spectral sequence for $M$ looks like

$\begin{array}{cccc}0 & 0 & 0 & 0 \\ 0 & E^{1,-1} & 0 & 0 \\ 0 & 0 & E^{2,-2} & E^{3,-2} \\ 0 & 0 & 0 & E^{3,-3}\end{array}$

Hence the filtration induced on $M$ by the spectral sequence looks like $M=F^{0} M=$ $F^{1} M \supset F^{2} M \supset \cdots$, so there is a surjective map

$$
M \rightarrow F^{1} M / F^{2} M=E_{\infty}^{1,-1}=\operatorname{ker}\left(E^{1,-1} \rightarrow E^{3,-2}\right) .
$$

This gives an exact sequence $M \rightarrow E^{1,-1} \rightarrow E^{3,-2}$. Since $\operatorname{dim}_{k}\left(E^{3,-2}\right)<\infty$, it follows that $\operatorname{GKdim}\left(E^{1,-1}\right) \leq 1$. If $E^{1,-1} \neq 0$, then $j\left(\operatorname{Ext}_{A}^{1}(M, A)\right)=1$ so, by Lemma 6.4, $\operatorname{GKdim} \underline{\operatorname{Ext}}_{A}^{1}(M, A)^{\vee}=2$; that is, $\operatorname{GKdim}\left(E^{1,-1}\right)=2$, which contradicts the foregoing. So we must have $E^{1,-1}=0$. Hence $E_{2}^{*,-1}=0$, so $\underline{\operatorname{Ext}}_{A}^{1}(M, A)=0$ and $j(M) \geq 2$. However, $j(M) \neq 3$ because $\operatorname{dim}_{k} M=\infty$, so $j(M)=2$.

Now suppose that GKdim $M=2$. By the first paragraph of the proof $j(M)$ is either 1 or 2 . Suppose that $j(M)=2$; we seek a contradiction. Let $\tau M$ be the sum of all finite dimensional graded submodules of $M$, and consider the exact sequence $0 \rightarrow \tau M \rightarrow M \rightarrow \bar{M} \rightarrow 0$. It follows easily (cf. [1], Prop. 2.46) that $\operatorname{Ext}_{A}^{i}(\bar{M}, A)=0$ for $i \neq 2$, so $\bar{M}$ is Cohen-Macaulay of depth one. But GKdim $\bar{M}=\mathrm{GKdim} M=2$ which contradicts Lemma 6.3.

Theorem 6.6. The algebra $A$ satisfies the Auslander condition: if $M$ is a finitely generated $A$-module and $N$ an $A$-submodule of $\operatorname{Ext}_{A}^{j}(M, A)$, then $\underline{\operatorname{Ext}}_{A}^{i}(N, A)=0$ for $i<j$.

Proof. Let $M$ be a non-zero finitely generated graded $A$-module, and $N$ a nonzero graded $A$-submodule of $\operatorname{Ext}_{A}^{i}(M, A)$. By Theorem 6.2, the $E_{2}$-page of the 
spectral sequence for $M$ looks like (6.2), so $j\left(\underline{\operatorname{Ext}}_{A}^{i}(M, A)\right) \geq i$. By Theorem 6.5, $\operatorname{GKdim}\left(\underline{\operatorname{Ext}}_{A}^{i}(M, A)\right) \leq 3-i$, so GKdim $N \leq 3-i$; by Theorem 6.5 applied to $N$, $j(N) \geq i$.

Corollary 6.7. The algebra $S$ satisfies the Auslander condition.

Proof. This follows from [11], Thm. 3.6.

Because $S$ satisfies the Auslander condition, the results in Sections 1 and 2 of [12] apply. In [12], Sect. 1, a non-zero module $M \in \operatorname{gr} S$ is said to be Cohen-Macaulay if its projective dimension is the smallest $i$ such that $\operatorname{Ext}_{S}^{i}(M, S)$ is non-zero. Since $S$ is Gorenstein, $M$ is Cohen-Macaulay in the sense of [12] if and only if it is CohenMacaulay in the sense of the present paper.

\section{Lines and rulings}

We continue to assume that $S$ and $A$ are as in the notation just before Proposition 5.3. We continue to use the notation $Q:=\operatorname{Proj} A$.

7.1. Line modules and maximal Cohen-Macaulay modules. A graded line module for $A$ or $S$ is a graded module $L$ that is cyclic and has Hilbert series

$$
H_{L}(t)=(1-t)^{-2} \text {. }
$$

We will write $\mathcal{O}_{L}$ for the image of $L$ in either Proj $A$ or Proj $S$. The class of $\mathcal{O}_{L}$ in $K_{0}(\operatorname{Proj} S)$ is $(1-t)^{2}$. By way of comparison, if $0 \neq x \in S_{1}$ and $\mathcal{O}_{H}$ denotes the image of $S / x S$ in Proj $S$, the class of $\mathcal{O}_{H}$ is $1-t$.

Lemma 7.1. Let $M \in M$. Then

(1) $\operatorname{Hom}_{\mathrm{Gr} A}(M(-1), A) \cong k^{2}$;

(2) if $0 \neq f \in \operatorname{Hom}_{\mathrm{Gr} A}(M(-1), A)$ then $f$ is injective, and

(3) coker $f$ is a line module.

Proof. (1) There is an exact sequence

$$
0 \rightarrow \underline{\operatorname{Hom}}_{A}(M, A) \rightarrow A^{2} \rightarrow \underline{\operatorname{Hom}}_{A}(\Omega M, A) \rightarrow 0
$$

of maximal Cohen-Macaulay left modules. Now $\underline{\operatorname{Hom}}_{A}(\Omega M, A)$ is indecomposable because $M$ is, and is obviously generated by its degree zero component which is 2dimensional because $\operatorname{Hom}_{\mathrm{Gr} A}(M, A)=0$. Hence $\underline{\operatorname{Hom}}_{A}(\Omega M, A)$ belongs to $\mathrm{M}^{\prime}$, the corresponding set of maximal Cohen-Macaulay left $A$-modules. By the left module version of Lemma 5.5, $\underline{\operatorname{Hom}}_{A}(M, A)(1)$ is also in $\mathrm{M}^{\prime}$, so $\operatorname{Hom}_{\mathrm{Gr} A}(M(-1), A) \cong$ $\underline{\operatorname{Hom}}_{A}(M, A)(1)_{0} \cong k^{2}$. 
(2) and (3): Because $M$ is 3-critical and $A$ is a domain, and hence 3-critical, every non-zero map $M(-1) \rightarrow A$ is injective. A Hilbert series computation shows that coker $f$ is a line module.

Proposition 7.2. Suppose that $S$ is a connected, graded, Gorenstein, Koszul algebra with Hilbert series $(1-t)^{-4}$. Let $z$ be a central regular element of degree two in $S$ and set $A=S /(z)$. Let $L$ be a line module for $A$. Then

(1) L has a linear resolution as an $S$-module;

(2) L has a linear resolution as an A-module, namely $\cdots \rightarrow A(-2)^{2} \rightarrow A(-1)^{2} \rightarrow$ $A \rightarrow L \rightarrow 0$

(3) L is Cohen-Macaulay of depth two and 2-critical with respect to GK-dimension;

(4) there is an exact sequence $0 \rightarrow M(-1) \rightarrow A \rightarrow L \rightarrow 0$ for a unique $M \in \mathbb{M}$;

(5) if $M$ and $L$ are as in part (4), then $F M \cong(F L)(1)$.

Proof. (1) By [12], Cor. 2.9, the minimal resolution of $L$ over $S$ is

$$
0 \rightarrow S(-2) \rightarrow S(-1)^{2} \rightarrow S \rightarrow L \rightarrow 0 .
$$

(2) If $L$ is any $A$-module having a linear resolution over $S$, then $L$ has a linear resolution over $A$ : to see this, use the fact that $\operatorname{Ext}_{S}^{1}(A, k) \cong k(2)$ and use the long exact sequence associated to the degenerate spectral sequence

$$
\underline{\operatorname{Ext}}_{A}^{p}\left(L, \underline{\operatorname{Ext}}_{S}^{q}(A, k)\right) \Rightarrow \underline{\operatorname{Ext}}_{S}^{p+q}(L, k) .
$$

This general fact for commutative rings is proved in [6].

(3) A line module is Cohen-Macaulay of depth two by [12], Prop. 2.8, and 2critical by [12], Cor. 1.11 .

(4) If $M(-1)$ is the kernel of a surjective map $A \rightarrow L$, then it follows from the long exact sequence for local cohomology that $M(-1)$ is Cohen-Macaulay of depth three. Every submodule of $A$ is indecomposable because $A$ is a domain. Hence $M(-1)$ is indecomposable. From the linear resolution of $L$, we see that $M(-1)$ is generated by $M(-1)_{1}$ and that $\operatorname{dim} M(-1)_{1}=2$, so $M \in \mathbb{M}$.

The uniqueness of $M$ will follow from (5) because if $M^{\prime}$ is another element of $M$ such that $M^{\prime} \cong \operatorname{ker}(A \rightarrow L)$, then $F M$ is isomorphic to $F M^{\prime}$ in $\mathrm{D}^{\mathrm{b}}\left(\right.$ qgr $\left.A^{!}\right)$, whence $M$ and $M^{\prime}$ are isomorphic in $\underline{\operatorname{MCM}}(A)$. Now apply Lemma 3.4.

(5) This follows from Lemma 3.3 because $M \cong \Omega L(1)$.

7.2. Rulings. For each $M \in \mathbb{M}$, we define the

ruling corresponding to $M:=\left\{L_{\phi}:=\operatorname{coker} \phi \mid \phi \in \mathbb{P}\left(\operatorname{Hom}_{\operatorname{Gr} A}(M(-1), A)\right)\right\}$.

Then

- each ruling consists of a $\mathbb{P}^{1}$ of line modules; 
- every line module belongs to a unique ruling;

- $Q$ has two rulings if it is smooth, and one otherwise.

The first of these facts follows from Lemma 7.1, the second from Proposition 7.2 (4), and the third is a consequence of Theorem 5.6 and the fact that the cardinality of $\mathrm{M}$ equals the number of isomorphism classes of simple $C(A)$-modules.

The results in the rest of this section provide further justification for using the word ruling.

Lemma 7.3. Let $L_{\phi}$ and $L_{\psi}\left(\phi, \psi \in \mathbb{P}^{1}\right)$ be lines in the same ruling. Then

(1) $L_{\phi} \cong L_{\psi}$ if and only if $\phi=\psi$;

(2) $\pi L_{\phi} \cong \pi L_{\psi}$ if and only if $\phi=\psi$.

Proof. (1) Let $L_{\phi}$ and $L_{\psi}$ be in the ruling corresponding to $M \in \mathbb{M}$. Because $M$ is indecomposable, $\operatorname{Hom}_{\mathrm{Gr} A}(M, M)$ is a local ring. It is finite dimensional and contains no non-zero nilpotents since $M$ is GK-homogeneous. Hence $\operatorname{Hom}_{\mathrm{Gr} A}(M, M) \cong k$. Because $L_{\phi}$ is cyclic, $L_{\phi} \cong L_{\psi}$ if and only $\operatorname{Im} \phi=\operatorname{Im} \psi$. However, the images are the same if and only if $\phi=\psi \theta$ for some $\theta \in \operatorname{Hom}_{\mathrm{Gr} A}(M(-1), M(-1))$; that is, if and only if $\phi=\psi$ as elements of $\mathbb{P}^{1}=\mathbb{P}\left(\operatorname{Hom}_{\mathrm{Gr} A}(M(-1), A)\right.$.

(2) Because $L_{\psi}$ is Cohen-Macaulay of depth two, the exact sequence (2.2) implies that $\omega \pi L_{\psi} \cong L_{\psi}$. Hence

$$
\operatorname{Hom}_{X}\left(\pi L_{\phi}, \pi L_{\psi}\right) \cong \operatorname{Hom}_{\mathrm{Gr} A}\left(L_{\phi}, \omega \pi L_{\psi}\right) \cong \operatorname{Hom}_{\mathrm{Gr} A}\left(L_{\phi}, L_{\psi}\right),
$$

so the result follows from (1).

The argument in (2) and the observation that each line module belongs to a unique ruling show that if $L$ and $L^{\prime}$ are line modules in different rulings, then $\pi L \not \pi L^{\prime}$.

Proposition 7.4. Let $L$ and $L^{\prime}$ be line modules.

(1) If $Q$ is smooth, then $L$ and $L^{\prime}$ belong to different rulings if and only if there is an exact sequence

$$
0 \rightarrow L^{\prime}(-1) \rightarrow A / a A \rightarrow L \rightarrow 0
$$

for some $0 \neq a \in A_{1}$.

(2) If $Q$ is not smooth there is always an exact sequence of the form (7.1).

Proof. There are exact sequences $0 \rightarrow M(-1) \rightarrow A \rightarrow L \rightarrow 0$ and $0 \rightarrow M^{\prime}(-1) \rightarrow$ $A \rightarrow L^{\prime} \rightarrow 0$ in which $M, M^{\prime} \in \mathbb{M}$.

(1) $\Longrightarrow$ : Suppose that $L$ and $L^{\prime}$ belong to different rulings; then $M \not M^{\prime}$, so $M^{\prime}(-1) \cong \Omega M$ by Corollary 5.7 . The first term in the exact sequence

$$
\operatorname{Hom}_{\mathrm{Gr} A}\left(A, L^{\prime}(-1)\right) \rightarrow \operatorname{Hom}_{\mathrm{Gr} A}\left(M(-1), L^{\prime}(-1)\right) \rightarrow \operatorname{Ext}_{\mathrm{Gr} A}^{1}\left(L, L^{\prime}(-1)\right) \rightarrow 0
$$


is zero, and $\operatorname{Hom}_{\operatorname{Gr} A}\left(M(-1), L^{\prime}(-1)\right)$ is isomorphic to

$$
\begin{aligned}
\operatorname{Ext}_{\mathrm{Gr} A}^{1}(M(-1), \Omega M(-1)) & \cong \operatorname{Hom}_{\underline{\underline{M C M}}}(M(-1), \Omega M(-1)[1]) \\
& =\operatorname{Hom}_{\underline{\underline{M C M}}}(M(-1), M(-1)) \\
& \cong k .
\end{aligned}
$$

Hence $\operatorname{Ext}_{\mathrm{Gr} A}^{1}\left(L, L^{\prime}(-1)\right) \neq 0$, and there is a non-split exact sequence

$$
0 \rightarrow L^{\prime}(-1) \rightarrow V \stackrel{\alpha}{\rightarrow} L \rightarrow 0
$$

in $\operatorname{Gr} A$. Choose $0 \neq \phi \in \operatorname{Hom}_{\mathrm{Gr} A}(A, V)$. The composition $\alpha \phi: A \rightarrow L$ is surjective because $L$ is cyclic, so $\operatorname{dim}_{k} \phi\left(A_{1}\right) \geq 2$. If $\operatorname{dim}_{k} \phi\left(A_{1}\right)=2$, then $L \cong$ $A / W A$, where $W=(\operatorname{ker} \phi)_{1}$, whence the map $V \rightarrow L$ splits, contrary to our assumption. Thus $\operatorname{dim}_{k} \phi\left(A_{1}\right)=3$. Hence there is some $0 \neq a \in A_{1}$ and a map $\psi: A / a A \rightarrow V$ that is surjective in degrees zero and one. Let $K=\operatorname{ker} \alpha \psi$. There is a commutative diagram

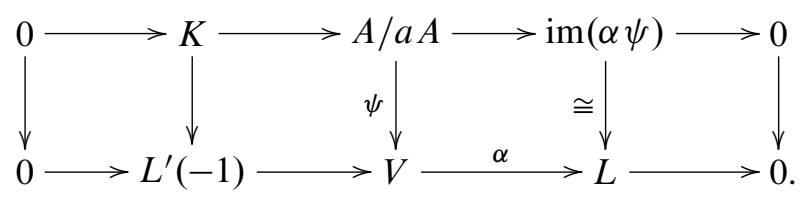

Since $\operatorname{dim}_{k}(\operatorname{im} \alpha \psi)_{1}<\operatorname{dim}_{k}(A / a A)_{1}, K_{1} \neq 0$, and hence the map $K \rightarrow L^{\prime}(-1)$ is surjective. It follows that $\psi$ is surjective, and hence injective because $V$ and $A / a A$ have the same Hilbert series. Hence we get a non-split exact sequence as claimed.

$\Longleftarrow$ : To show that $L^{\prime}$ is in a different ruling from $L$ it suffices to show that $\operatorname{Ext}_{\mathrm{Gr} A}^{1}\left(L^{\prime}, M(-1)\right)=0$.

Apply $\operatorname{Hom}_{\mathrm{Gr} A}(-, M(-1))$ to the exact sequence (7.1). A computation shows that $\operatorname{Ext}_{\mathrm{Gr} A}^{1}(A / a A(1), M(-1))=0$. Since $\operatorname{Ext}_{\mathrm{Gr} A}^{2}(A / a A(1), M(-1))=0$, we have $\operatorname{Ext}_{\operatorname{Gr} A}^{1}\left(L^{\prime}, M(-1)\right) \cong \operatorname{Ext}_{\mathrm{Gr} A}^{2}(L(1), M(-1))$. From the exact sequence $0 \rightarrow$ $M \rightarrow A(1) \rightarrow L(1) \rightarrow 0$, we see that

$$
\operatorname{Ext}_{\mathrm{Gr} A}^{2}(L(1), M(-1)) \cong \operatorname{Ext}_{\mathrm{Gr} A}^{1}(M, M(-1)) \cong \operatorname{Hom}_{\underline{\mathrm{MCM}}}(M, M(-1)[1])
$$

and this is zero as we see by applying the functor $F$.

(2) The proof of the implication $\Longrightarrow$ works when $Q$ is not smooth too because then $M^{\prime} \cong M \cong \Omega M(1)$.

\section{Points on quadrics}

We continue to assume that $S$ and $A$ are as in the notation just before Proposition 5.3. We also assume $Q$ is smooth. 
8.1. Point modules. A graded point module for $A$ or $S$ is a graded module $P$ that is cyclic and has Hilbert series

$$
H_{P}(t)=(1-t)^{-1} \text {. }
$$

We will write $\mathcal{O}_{P}$ for the image of $P$ in either Proj $A$ or Proj $S$. The class of $\mathcal{O}_{P}$ in $K_{0}(\operatorname{Proj} S)$ is $(1-t)^{3}$.

Lemma 8.1. Let $M \in M$. If $L_{\phi}$ is in the ruling corresponding to $\Omega M(1)$, there is an exact sequence of the form

$$
0 \rightarrow A \rightarrow M \rightarrow L_{\phi} \rightarrow 0 .
$$

Proof. By hypothesis, there is an exact sequence

$$
0 \rightarrow \Omega M \stackrel{\phi}{\rightarrow} A \stackrel{\bar{\phi}}{\rightarrow} L_{\phi} \rightarrow 0
$$

There is also an exact sequence

$$
0 \rightarrow \Omega M \stackrel{\theta}{\rightarrow} A^{2} \rightarrow M \rightarrow 0 .
$$

Since $M$ is maximal Cohen-Macaulay $\operatorname{Ext}_{\mathrm{Gr} A}^{1}(M, A)=0$, so the natural map

$$
\operatorname{Hom}_{\mathrm{Gr} A}\left(A^{2}, A\right) \rightarrow \operatorname{Hom}_{\mathrm{Gr} A}(\Omega M, A), \quad \rho \mapsto \rho \circ \theta,
$$

is surjective and hence an isomorphism because $\operatorname{Hom}_{\mathrm{Gr} A}(M, A)=0$. Hence $\phi=\rho \theta$ for a unique $\rho: A^{2} \rightarrow A$.

The map $\bar{\phi} \rho$ in the diagram

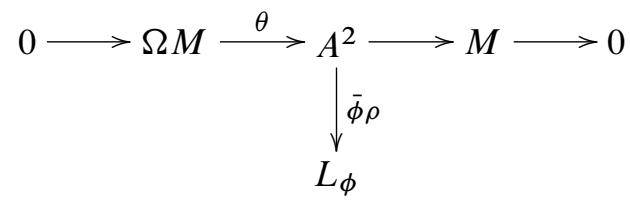

is surjective because $\bar{\phi}$ and $\rho$ are, and $\bar{\phi} \rho \theta=\bar{\phi} \phi=0$, so there is a surjective map $\psi: M \rightarrow L_{\phi}$.

Now $(\operatorname{ker} \psi)_{0} \neq 0$ because $\operatorname{dim} M_{0}>\operatorname{dim}\left(L_{\phi}\right)_{0}$, so there is a non-zero map $A \rightarrow \operatorname{ker} \psi$. Since $M$ is 3-critical so is $\operatorname{ker} \psi$, so the map $A \rightarrow \operatorname{ker} \psi$ is injective. But

$$
\mathrm{H}_{\mathrm{ker} \psi}(t)=H_{M}(t)-H_{L_{\phi}}(t)=H_{A}(t),
$$

so the map $A \rightarrow \operatorname{ker} \psi$ must be an isomorphism.

Proposition 8.2. Let $M \in M$. If $L_{\phi}$ is in the ruling corresponding to $\Omega M(1)$, there is an exact sequence

$$
0 \rightarrow L(-1) \rightarrow L_{\phi} \rightarrow P \rightarrow 0
$$

in which $P$ is a point module and $L$ is a line module in the same ruling as $L_{\phi}$. 
Proof. By hypothesis, there is an exact sequence of the form (8.2).

Claim: $\operatorname{dim} \operatorname{Hom}_{\mathrm{Gr} A}\left(M(-1), L_{\phi}\right) \geq 2$.

Proof. Applying $\operatorname{Hom}_{\mathrm{Gr} A}(M(-1),-)$ to $(8.2)$ yields an exact sequence

$$
\operatorname{Hom}_{\mathrm{Gr} A}(M(-1), A) \stackrel{\gamma}{\rightarrow} \operatorname{Hom}_{\mathrm{Gr} A}\left(M(-1), L_{\phi}\right) \stackrel{\delta}{\rightarrow} \operatorname{Ext}_{\mathrm{Gr} A}^{1}(M(-1), \Omega M) .
$$

If $Q$ is smooth, then $\operatorname{Hom}_{\mathrm{Gr} A}(M(-1), \Omega M)=0$ by Corollary 5.7, so $\gamma$ is injective, and the claim follows from Lemma 7.1.

Suppose that $Q$ is not smooth. Then $M(-1) \cong \Omega M$ by Corollary 5.7 ; thus $\operatorname{Hom}_{\mathrm{Gr} A}(M(-1), \Omega M) \cong k$ by the argument in the proof of Lemma 7.3 , and $\operatorname{Ext}_{\mathrm{Gr} A}^{1}(M(-1), \Omega M) \neq 0$ by the proof of Step 1 in Theorem 5.6. However, $\operatorname{Ext}_{\mathrm{Gr} A}^{1}(M(-1), A)=0$, so the claim holds in this case too.

The restriction of each non-zero $\psi \in \operatorname{Hom}_{\mathrm{Gr} A}\left(M(-1), L_{\phi}\right)$ gives a non-zero map $M(-1)_{1} \rightarrow\left(L_{\phi}\right)_{1}$ between two 2 -dimensional vector spaces. Now every line in the projective space $\mathbb{P}^{3}=\mathbb{P}\left(\operatorname{Hom}_{k}\left(k^{2}, k^{2}\right)\right)$ meets the quadric of singular maps, so there is a non-zero $\psi$ such that $(\operatorname{ker} \psi)_{1} \neq 0$. There is a non-zero map $A(-1) \rightarrow \operatorname{ker} \psi$; this map is injective because $A(-1)$ and $\operatorname{ker} \psi$ are 3-critical; the cokernel of the composition $\alpha: A(-1) \rightarrow \operatorname{ker} \psi \rightarrow M(-1)$ is cyclic because $\operatorname{dim} M_{0}=1+$ $\operatorname{dim} A(-1)_{1}$ and $M$ is generated by $M_{0}$; the Hilbert series of coker $\alpha$ is

$$
H_{M(-1)}(t)-H_{A(-1)}(t)=t(1-t)^{-2},
$$

so coker $\alpha$ is a shifted line module, say $L(-1)$.

Because $\psi \alpha=0$, it follows from the diagram

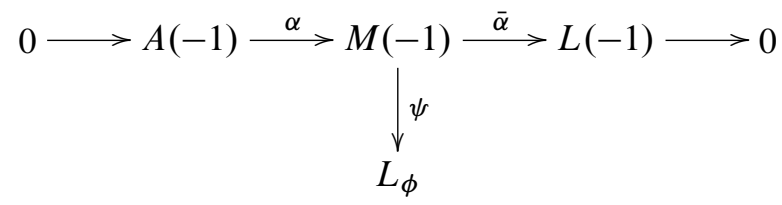

that $\psi=\beta \bar{\alpha}$ for some $\beta: L(-1) \rightarrow L_{\phi}$. Because $L(-1)$ and $L_{\phi}$ are 2-critical $\beta$ is injective, and coker $\beta$ is cyclic with Hilbert series $(1-t)^{-2}-t(1-t)^{-2}$. Hence $\operatorname{coker} \beta$ is a point module.

It remains only to show that $L$ is in the same ruling as $L_{\phi}$. Consider the diagram

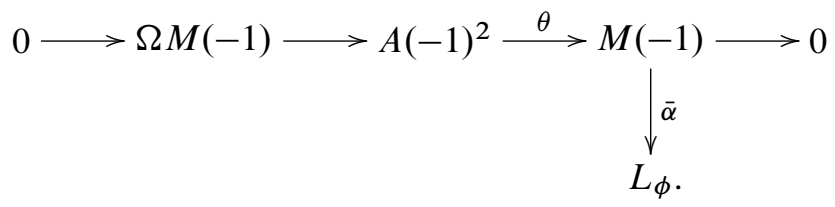

Since $L$ is cyclic, the restriction of $\bar{\alpha} \theta$ to one of the copies of $A(-1)$ is surjective, so, after a Hilbert series computation, we see that the kernel of $\bar{\alpha} \theta$ must be isomorphic to $\Omega M(-1)$. From the exact sequence $0 \rightarrow \Omega M(-1) \rightarrow A(-1) \rightarrow L(-1) \rightarrow 0$ we see that $L$ is in the same ruling as $L_{\phi}$. 
Lemma 8.3. Given a line module $L$, there is an exact sequence of the form

$$
0 \rightarrow L(-1) \rightarrow L_{\phi} \rightarrow P \rightarrow 0
$$

in which $P$ is a point module and $L_{\phi}$ is a line module in the same ruling as $L$.

Proof. This follows from duality and Proposition 8.2 for left modules.

Since $L$ is Cohen-Macaulay of depth two, $\operatorname{Ext}_{A}^{1}(L, A)$ is a left line module. By Proposition 8.2 for left modules, there is an exact sequence

$$
0 \rightarrow L^{\prime}(-1) \rightarrow \underline{\operatorname{Ext}}_{A}^{1}(L, A) \rightarrow P^{\prime} \rightarrow 0
$$

where $L^{\prime}$ and $P^{\prime}$ are a left line and point module, respectively. Since $P^{\prime}$ is CohenMacaulay of depth one, applying $\underline{\operatorname{Hom}}_{A}(-, A)$ to this exact sequence gives an exact sequence

$$
0 \rightarrow \underline{\operatorname{Ext}}_{A}^{1}\left(\underline{\operatorname{Ext}}_{A}^{1}((L, A), A) \rightarrow \underline{\operatorname{Ext}}_{A}^{1}\left(L^{\prime}, A\right)(1) \rightarrow \underline{\operatorname{Ext}}_{A}^{1}\left(P^{\prime}, A\right) \rightarrow 0 .\right.
$$

Twisting this by $(-1)$ gives the desired exact sequence (8.3).

\section{The Grothendieck group of a smooth quadric}

We continue to assume that $S$ and $A$ are as in the notation just before Proposition 5.3. We also assume that $Q$ is smooth. We will write $C$ for the algebra $C(A)$ that is isomorphic to $M_{2}(k) \oplus M_{2}(k)$.

We write $K_{0}(\mathrm{~A})$ for the Grothendieck group of an abelian category A and define $K_{0}(Q):=K_{0}(\operatorname{coh} Q)$. We will show that there is an isomorphism $K_{0}(Q) \cong$ $K_{0}\left(\mathbb{P}^{1} \times \mathbb{P}^{1}\right)$ of abelian groups that is compatible with the Euler forms. However, the discussion after Theorem 10.2 shows that the effective cones need not coincide under this isomorphism.

9.1. The localization sequence for K-theory gives the exact rows in the diagram

$$
\begin{gathered}
K_{0}\left(\operatorname{fdim} A^{!}\right) \stackrel{\alpha}{\longrightarrow} K_{0}\left(\operatorname{gr} A^{!}\right) \stackrel{\beta}{\longrightarrow} K_{0}(\bmod C) \longrightarrow 0 \\
\phi \mid \cong \\
K_{0}(\operatorname{fdim} A) \longrightarrow K_{0}(\operatorname{gr} A) \longrightarrow K_{0}(Q) \longrightarrow .
\end{gathered}
$$

The isomorphism $\phi$ is induced by the Koszul duality functor

$$
K: \mathrm{D}^{\mathrm{b}}(\operatorname{gr} A) \rightarrow \mathrm{D}^{\mathrm{b}}\left(\operatorname{gr} A^{!}\right)
$$

and the fact that $K_{0}(\mathrm{~A}) \cong K_{0}\left(\mathrm{D}^{\mathrm{b}}(\mathrm{A})\right)$. Thus $\phi([N])=[K N]$ is an isomorphism of abelian groups. 
We use the degree shift functors $( \pm 1)$ on the categories fdim $A^{!}, \operatorname{gr} A^{!}, \operatorname{fdim} A$, $\operatorname{gr} A$, and $\bmod Q$, to make their Grothendieck groups into $\mathbb{Z}\left[t, t^{-1}\right]$-modules via

$$
t \cdot[M]=[M(-1)] .
$$

Exact functors between these categories that "commute" with the shift functors induce $\mathbb{Z}\left[t, t^{-1}\right]$-module homomorphisms between their Grothendieck groups.

Let $M$ and $M^{\prime}$ be the two indecomposable maximal Cohen-Macaulay modules such that $\mathrm{M}=\left\{M(1), M^{\prime}(1)\right\}$.

We will use the notation

$$
a=[A], \quad m=[M], \quad m^{\prime}=\left[M^{\prime}\right], \quad \ell:=a-m, \quad \ell^{\prime}:=a-m^{\prime}
$$

for these elements of $K_{0}(\bmod A)$. We will use the same notation for the images of $a$, $m, m^{\prime}, \ell$ and $\ell^{\prime}$ in $K_{0}(Q)$ and will always take care to indicate which Grothendieck group we are working in.

The line modules $L$ and $L^{\prime}$ in the rulings determined by $M$ and $M^{\prime}$ respectively occur in exact sequences of the form $0 \rightarrow M \rightarrow A \rightarrow L \rightarrow 0$ and $0 \rightarrow M^{\prime} \rightarrow A \rightarrow$ $L^{\prime} \rightarrow 0$, so all the line modules in a single ruling give the same class in $K_{0}(\bmod A)$, namely $[L]=\ell=a-m$ and $\ell^{\prime}:=\left[L^{\prime}\right]=a-m^{\prime}$, respectively.

Proposition 9.1. The Grothendieck group of $Q$ is free of rank 4 with basis

$$
\left\{a, m, m^{\prime}, a t\right\}
$$

and the action of $\mathbb{Z}\left[t, t^{-1}\right]$ on it is given by

$$
\begin{aligned}
m t & =2 a t-m^{\prime} \\
m^{\prime} t & =2 a t-m, \\
a t^{2} & =a(1+4 t)-2\left(m+m^{\prime}\right) .
\end{aligned}
$$

As an R-module,

$$
K_{0}(Q) \cong \frac{R a \oplus R \ell}{\left(\ell(1-t)^{2}, a\left(1-t^{2}\right)-2 \ell(1-t)\right)} .
$$

Proof. Write $R=\mathbb{Z}\left[t, t^{-1}\right]$ and $C=A^{!}\left[w^{-1}\right]_{0}$.

By Dévissage, $K_{0}\left(\right.$ fdim $\left.A^{!}\right) \cong K_{0}(\bmod k)$. Taking Hilbert series gives a map $K_{0}\left(\bmod A^{!}\right) \rightarrow \mathbb{Z}[[t]]\left[t^{-1}\right]$; since $K_{0}(\bmod k) \cong \mathbb{Z}\left[t, t^{-1}\right]$ it follows that the map $\alpha$ in (9.1) is injective. Since $Q$ is smooth, $K_{0}(\bmod C)=\mathbb{Z}\left[S_{1}\right] \oplus \mathbb{Z}\left[S_{2}\right] \cong \mathbb{Z}^{2}$ where $S_{1}$ and $S_{2}$ are the two simple $C$-modules; hence the top row of (9.1) splits, and

$$
K_{0}\left(\bmod A^{!}\right)=R[k] \oplus \mathbb{Z}\left[\tilde{S}_{1}\right] \oplus \mathbb{Z}\left[\widetilde{S}_{2}\right]
$$

where $\widetilde{S}_{1}$ and $\widetilde{S}_{2}$ are the liftings of $S_{1}$ and $S_{2}$ via the functor $-\otimes_{C} A^{!}\left[w^{-1}\right]_{\geq 0}$. Transferring this to $A$ via Koszul duality, we see that

$$
K_{0}(\bmod A)=R a \oplus \mathbb{Z} m \oplus \mathbb{Z} m^{\prime} .
$$


From the exact sequences in Corollary 5.8, we obtain relations

$$
2 a t=m t+m^{\prime}=m^{\prime} t+m
$$

in $K_{0}(\bmod A)$. Hence there is a surjective map

$$
\frac{R a \oplus R m}{(2 a t-m-(2 a t-m t) t)} \rightarrow K_{0}(\bmod A)
$$

of $\mathbb{Z}\left[t, t^{-1}\right]$-modules. However, it follows from (9.2) that there is also a surjective map

$$
K_{0}(\bmod A)=R a \oplus \mathbb{Z} m \oplus \mathbb{Z} m^{\prime} \rightarrow \frac{R a \oplus R m}{(2 a t-m-(2 a t-m t) t)},
$$

so we conclude that (9.3) is an isomorphism.

Since $\ell=a-m$, we also have

$$
K_{0}(\bmod A) \cong \frac{R a \oplus R \ell}{\left(a(1-t)^{2}-\ell\left(1-t^{2}\right)\right)} .
$$

Now $K_{0}(\operatorname{fdim} A) \cong K_{0}(\bmod k) \cong \mathbb{Z}\left[t, t^{-1}\right]$ with basis $[k] \leftrightarrow 1$, so

$$
K_{0}(Q) \cong K_{0}(\bmod A) /([k]),
$$

where $([k])$ denotes the $\mathbb{Z}\left[t, t^{-1}\right]$-submodule generated by $[k]$.

We now compute $[k]$. From the Hilbert series for $A^{!}$, we see that the truncated minimal resolution of $k$ looks like

$$
0 \rightarrow N \rightarrow A(-2)^{7} \rightarrow A(-1)^{4} \rightarrow A \rightarrow k \rightarrow 0 .
$$

It is clear that $N$ is a maximal Cohen-Macaulay module and that $N(3)$ has a linear resolution.

Let $F: \bmod A \rightarrow \mathrm{D}^{\mathrm{b}}\left(\mathrm{qgr} A^{!}\right)$be the functor in Lemma 3.3. Since $N \cong \Omega^{3} k$, that lemma shows that

$$
F(N(3)) \cong(F k)(3) \cong A^{!}(3) .
$$

The equivalence qgr $A^{!} \rightarrow \bmod C$ sends $A^{!}$to $C$. The degree twist (1) on qgr $A^{!}$ induces an auto-equivalence of $\bmod C$, but every auto-equivalence of $\bmod C$ sends ${ }_{C} C$ to ${ }_{C} C$. Thus, if $G$ is the composition

$$
\bmod A \stackrel{F}{\rightarrow} \mathrm{D}^{\mathrm{b}}\left(\mathrm{qgr} A^{!}\right) \stackrel{\sim}{\longrightarrow} \mathrm{D}^{\mathrm{b}}(\bmod C),
$$

then

$$
G(N(3)) \cong C(3) \cong C .
$$

The functor $G$ sends the two maximal Cohen-Macaulay modules $M(1)$ and $M^{\prime}(1)$ to the two simple left $C$-modules, so we see that

$$
G(N(3)) \cong G\left(M(1)^{\oplus 2} \oplus M^{\prime}(1)^{\oplus 2}\right) .
$$


It now follows from Buchweitz's duality (Theorem 3.2) that

$$
N \cong M(-2)^{\oplus 2} \oplus M^{\prime}(-2)^{\oplus 2} \text {. }
$$

The truncated resolution of $k$ and equation (9.2) therefore give

$$
[k]=\left(1-4 t+7 t^{2}\right) a-\left(2 m+2 m^{\prime}\right) t^{2}=\left(1+4 t-t^{2}\right) a-2\left(m+m^{\prime}\right) .
$$

Hence, in $K_{0}(Q), a t^{2}=a(1+4 t)-2\left(m+m^{\prime}\right)$. It follows that $K_{0}(Q)$ has basis $\left\{a, m, m^{\prime}, a t\right\}$, as claimed.

By Proposition 8.2, $A$ has some graded point modules, so we define

$$
p:=\ell(1-t) \text { and } p^{\prime}:=\ell^{\prime}(1-t)
$$

for the corresponding classes in $K_{0}(Q)$. By $(9.2)\left(m-m^{\prime}\right)(1-t)=0$, so

$$
p=\ell(1-t)=(a-m)(1-t)=\left(a-m^{\prime}\right)(1-t)=\ell^{\prime}(1-t)=p^{\prime} .
$$

Proposition 9.2. The sets $\left\{a, m, m^{\prime}, p\right\}$ and $\left\{a, \ell, \ell^{\prime}, p\right\}$ provide $\mathbb{Z}$-bases for $K_{0}(Q)$. The $\mathbb{Z}\left[t, t^{-1}\right]$-action is given by

$$
a(1-t)=\ell+\ell^{\prime} t=\ell^{\prime}+\ell t, \quad \ell(1-t)=\ell^{\prime}(1-t)=p, \quad p(1-t)=0 .
$$

Proof. Recall that $\ell=a-m$ and $\ell^{\prime}=a-m^{\prime}$, so $\left\{a, \ell, \ell^{\prime}, a t\right\}$ is a basis for $K_{0}(Q)$. Furthermore,

$$
p=\ell(1-t)=(a-m)(1-t)=a-a t-m+\left(2 a t-m^{\prime}\right)=a+a t-m-m^{\prime},
$$

and it follows from this that the two claimed bases are indeed bases for $K_{0}(Q)$. The action of $t$ is already implicit, if not explicit, in the calculations made in the proof of Proposition 9.1.

The annihilator of $K_{0}(Q)$ as a $\mathbb{Z}\left[t, t^{-1}\right]$-module is $(1-t)^{3}$. The submodule of $K_{0}(Q)$ annihilated by $(1-t)$ is $\mathbb{Z} p \oplus \mathbb{Z}\left(\ell-\ell^{\prime}\right)$.

Taking Hilbert series gives a $\mathbb{Z}\left[t, t^{-1}\right]$-module homomorphism $K_{0}(\bmod A) \rightarrow$ $\mathbb{Z}\left[t, t^{-1},(1-t)^{-1}\right],[N] \mapsto H_{N}(t)$. Likewise there is a homomorphism $q: K_{0}(\bmod A) \rightarrow \mathbb{Z}\left[t, t^{-1}\right]$ defined by

$$
q[N]=H_{N}(t)(1-t)^{3} .
$$

Because $q[k]=(1-t)^{3}$, there is an induced $\mathbb{Z}\left[t, t^{-1}\right]$-module homomorphism

$$
\bar{q}: K_{0}(Q) \rightarrow \mathbb{Z}\left[t, t^{-1}\right] /(1-t)^{3} .
$$

One has

$$
\bar{q}(a)=1+t, \quad \bar{q}(\ell)=\bar{q}\left(\ell^{\prime}\right)=1-t, \quad \bar{q}(p)=(1-t)^{2} .
$$

Suppose that $N \in \bmod A$ has GK-dimension one. Then $H_{N}(t)=f(t)(1-t)^{-1}$ for some $f(t) \in \mathbb{Z}\left[t, t^{-1}\right]$, so $\bar{q}[\pi N]$ belongs to the ideal of $\mathbb{Z}\left[t, t^{-1}\right] /(1-t)^{3}$ generated by $(1-t)^{2}$. It follows that

$$
[\pi N] \in \mathbb{Z} p \oplus \mathbb{Z}\left(\ell-\ell^{\prime}\right) .
$$


9.2. The Euler form. The Euler form on $K_{0}(Q)$ is denoted by $(-,-)$ and is defined by

$$
([M],[N])=\sum_{i=0}^{2}(-1)^{i} \operatorname{dim}_{k} \operatorname{Ext}_{Q}^{i}(M, N)
$$

Proposition 9.3. The Euler form on $K_{0}(Q)$ is given by

$$
(a, a)=(a, \ell)=\left(a, \ell^{\prime}\right)=(a, p)=(p, a)=1 ; \quad(\ell, a)=\left(\ell^{\prime}, a\right)=-1
$$

and

$$
(\ell, \ell)=\left(\ell^{\prime}, \ell^{\prime}\right)=(\ell, p)=\left(\ell^{\prime}, p\right)=(p, \ell)=\left(p, \ell^{\prime}\right)=(p, p)=0
$$

and

$$
\left(\ell, \ell^{\prime}\right)=\left(\ell^{\prime}, \ell\right)=-1
$$

Proof. Let $P$ be a graded point module occurring in an exact sequence of the form $0 \rightarrow L_{\phi}(-1) \rightarrow L_{\psi} \rightarrow P \rightarrow 0$ where $L_{\psi}$ and $L_{\phi}$ are line modules in the same ruling. From the Cohen-Macaulayness of $A, M, M^{\prime}, P$ we see that

$$
(a, a)=1, \quad(a, m)=\left(a, m^{\prime}\right)=0, \quad(a, p)=1,
$$

whence

$$
(a, a)=(a, \ell)=\left(a, \ell^{\prime}\right)=(a, p)=1 .
$$

Serre duality on $Q$ takes the form $\operatorname{Ext}_{Q}^{i}(\mathcal{F}, \mathcal{E}) \cong \operatorname{Ext}_{Q}^{2-i}(\mathcal{E}, \mathcal{F}(-2))^{*}$ for $\mathcal{F}, \mathcal{E} \in$ $\bmod Q$. Hence $(x, y)=\left(y, x t^{2}\right)$ for all $x, y \in K_{0}(Q)$. Also, $(x t, y t)=(x, y)$.

We have $(\ell, a)=\left(a, \ell t^{2}\right)=(a, \ell-2 p)=-1$, and similarly, $\left(\ell^{\prime}, a\right)=-1$. Also, $(p, a)=\left(a, p t^{2}\right)=(a, p)=1$. In summary,

$$
(\ell, a)=\left(\ell^{\prime}, a\right)=-1, \quad(p, a)=1 .
$$

Now we show that $(m, m)=1$. The first step is to show that $\operatorname{Ext}_{Q}^{1}(\mathcal{M}, \mathcal{M})=0$. If $0 \rightarrow \mathcal{M} \rightarrow \mathcal{F} \rightarrow \mathcal{M} \rightarrow 0$ is exact, then applying $\omega$ gives an exact sequence $0 \rightarrow M \rightarrow F \rightarrow M \rightarrow 0$ because $R^{1} \omega M=H_{\mathrm{m}}^{2}(M)=0$ and $\omega \pi M \cong M$. But $\operatorname{Ext}_{\mathrm{Gr} A}^{1}(M, M) \cong \operatorname{Hom}_{\mathrm{MCM}}(M, M[1])=0$, where the last equality follows by applying the functor $F$, so the sequence in $\operatorname{Gr} A$ splits; but the original sequence in Qcoh $Q$ is obtained by applying $\omega$ to this split sequence, so it splits too. Hence $\operatorname{Ext}_{Q}^{1}(\mathcal{M}, \mathcal{M})=0$. Now, $\operatorname{Ext}_{Q}^{2}(\mathcal{M}, \mathcal{M}) \cong \operatorname{Hom}_{Q}(\mathcal{M}, \mathcal{M}(-2))^{*} \cong$ $\operatorname{Hom}_{\mathrm{Gr} A}(M, M(-2))^{*}=0$ because $M(-2)_{1}=0$. Finally, $\operatorname{Hom}_{Q}(\mathcal{M}, \mathcal{M}) \cong$ $\operatorname{Hom}_{\mathrm{Gr} A}(M, M)=k$, so $(m, m)=1$.

Using this gives

$(\ell, \ell)=(a-m, a-m)=(a, a-m)-(m, a)+(m, m)=1-(a-\ell, a)+1=0$,

and similarly, $\left(\ell^{\prime}, \ell^{\prime}\right)=0$. Using Serre duality, we obtain

$$
0=(\ell, \ell)=\left(\ell, \ell t^{2}\right)=(\ell, \ell-2 p)=-2(\ell, p),
$$


which gives $(\ell, p)=0$. Therefore

$$
\left(\ell, \ell^{\prime}\right)=\left(\ell, \ell^{\prime}+\ell-p\right)=\left(\ell, \ell^{\prime}+\ell t\right)=(\ell, a(1-t))=-1-(\ell, a t)
$$

and hence

$$
\left(\ell, \ell^{\prime}\right)=-1-\left(a t, \ell t^{2}\right)=-1-(a, \ell t)=-1-(a, \ell-p)=-1 .
$$

Similarly, $\left(\ell^{\prime}, \ell\right)=-1$.

Finally,

$$
(p, p)=(\ell(1-t), p)=-(\ell t, p)=-\left(\ell, p t^{-1}\right)=-(\ell, p)=0
$$

and

$$
(p, \ell)=\left(\ell, p t^{2}\right)=(\ell, p)=0 .
$$

This completes the proof.

Proposition 9.3 is exactly as in the commutative case - of course, our proof applies to that case too.

9.3. The intersection pairing on $Q$. Recall that $\ell$ and $\ell^{\prime}$ are the classes in $K_{0}(Q)$ of $\mathcal{O}_{L}$ and $c O_{L^{\prime}}$ where $L$ and $L^{\prime}$ are line modules belonging to different rulings.

The interpretation of the equality $\left(\ell, \ell^{\prime}\right)=-1$ in Proposition 9.3 is that a line in one ruling meets a line in the other ruling with multiplicity one. In the commutative case this means the two lines span a hyperplane. Proposition 7.4 (1) is the appropriate analogue of this. It is therefore sensible to introduce the notation

$$
h:=\left[\mathcal{O}_{Q}\right]-\left[\mathcal{O}_{Q}(-1)\right]=\ell+\ell^{\prime} t=\ell^{\prime}+\ell t .
$$

We now define an intersection pairing on $K_{0}(Q)$ by

$$
b \cdot c:=-(b, c) .
$$

The next calculation shows that everything behaves as it does for points and lines on a smooth quadric surface in $\mathbb{P}^{3}$, i.e., as for $\mathbb{P}^{1} \times \mathbb{P}^{1}$.

Proposition 9.4. The intersection pairing has the following properties:

$$
\begin{array}{r}
\ell \cdot \ell=\ell \cdot p=h \cdot p=p \cdot h=p \cdot \ell=\ell^{\prime} \cdot \ell^{\prime}=0 ; \\
\ell \cdot \ell^{\prime}=\ell \cdot h=\ell^{\prime} \cdot h=h \cdot \ell^{\prime}=h \cdot \ell=1 ; \\
\ell \cdot \ell^{\prime}=\ell^{\prime} \cdot \ell=1 .
\end{array}
$$

Proof. The calculations are as follows:

$$
\begin{aligned}
& (h, \ell)=\left(\ell+\ell^{\prime} t, \ell\right)=\left(\ell^{\prime} t, \ell\right)=\left(\ell^{\prime}-p, \ell\right)=-1 ; \\
& (h, p)=\left(\ell+\ell^{\prime} t, p\right)=(\ell, p)+\left(\ell^{\prime}, p t^{-1}\right)=0 ; \\
& (\ell, h)=\left(h, \ell t^{2}\right)=(h, \ell-2 p)=-1 ;
\end{aligned}
$$

and $(p, h)=\left(h, p t^{2}\right)=(h, p)=0$. 
One other computation of interest is $\left(m, m^{\prime}\right)=0$.

Proposition 9.5. Suppose that $Q$ is smooth. Let $L$ and $L^{\prime}$ be non-isomorphic line modules for $A$, and $\mathcal{O}_{L}$ and $\mathcal{O}_{L^{\prime}}$ their images in Proj $Q$. The following are equivalent:

(1) $\operatorname{Ext}_{Q}^{1}\left(\mathcal{O}_{L}, \mathcal{O}_{L^{\prime}}\right)=0$;

(2) $\operatorname{Ext}_{Q}^{i}\left(\mathcal{O}_{L}, \mathcal{O}_{L^{\prime}}\right)=0$ for all $i$;

(3) $L$ and $L^{\prime}$ belong to the same ruling.

Proof. Because $L^{\prime}$ is Cohen-Macaulay of depth $2, \omega \pi L^{\prime} \cong L^{\prime}$. Hence

$$
\operatorname{Hom}_{Q}\left(\mathcal{O}_{L}, \mathcal{O}_{L^{\prime}}\right) \cong \operatorname{Hom}_{\mathrm{Gr} A}\left(L, \omega \pi L^{\prime}\right) \cong \operatorname{Hom}_{\mathrm{Gr} A}\left(L, L^{\prime}\right)=0 .
$$

By Serre duality, $\operatorname{Ext}_{Q}^{2}\left(\mathcal{O}_{L}, \mathcal{O}_{L^{\prime}}\right) \cong \operatorname{Hom}_{Q}\left(\mathcal{O}_{L^{\prime}}, \mathcal{O}_{L}(-2)\right)$; because $L$ is CohenMacaulay of depth 2, this is isomorphic to $\operatorname{Hom}_{\mathrm{Gr} A}\left(L^{\prime}, L(-2)\right)$, which is zero because $L(-2)_{0}=0$. Hence $(1) \Longleftrightarrow(2)$.

By Proposition 9.3, $L$ and $L^{\prime}$ belong to the same ruling if and only if $\left(\left[\mathcal{O}_{L}\right],\left[\mathcal{O}_{L^{\prime}}\right]\right)=0$. This, together with the observations in the previous paragraph, shows that (3) is equivalent to (1) and (2).

If $Q$ is not smooth, then $\operatorname{Ext}_{Q}^{1}\left(\mathcal{O}_{L}, \mathcal{O}_{L^{\prime}}\right) \neq 0$, and if $L \not L^{\prime}$, then $\operatorname{Ext}_{Q}^{2}\left(\mathcal{O}_{L}, \mathcal{O}_{L^{\prime}}\right)$ and $\operatorname{Hom}_{Q}\left(\mathcal{O}_{L}, \mathcal{O}_{L^{\prime}}\right)$ are both zero.

\section{The Sklyanin quadrics}

Throughout this section $S$ denotes a 4-dimensional Sklyanin algebra and

$$
\mathbb{P}_{\text {Skly }}^{3}=\operatorname{Proj} S \text {. }
$$

We recall some results from [12], [15], [16], [18], [22], and [23].

10.1. The data used to define $S$ is a triple $(E, \mathscr{L}, \tau)$ consisting of an elliptic curve $E$, a degree four line bundle $\mathscr{L}$ on it, a translation automorphism $\tau$ of $E$, and $S$ is a quotient of the tensor algebra on $H^{0}(E, \mathscr{L})$ having Hilbert series $(1-t)^{-4}$. Like the polynomial ring, $S$ is Gorenstein, and its dualizing module is $\omega_{S} \cong S(-4)$ as a one-sided $S$-module. Furthermore, $S$ is a noetherian domain and a Koszul algebra. Thus $\mathbb{P}_{\text {Skly }}^{3}$ is a quantum $\mathbb{P}^{3}$ in the sense of Section 2.8.

Because $S_{1}=H^{0}(E, \mathscr{L})$ we can, and will, consider $E$ as a fixed quartic curve in $\mathbb{P}\left(S_{1}^{*}\right)$. We fix an identity element 0 for $E$ such that four points of $E$ are coplanar if and only if their sum is 0 . We therefore identify $\tau$ with a point on $E$, so the translation automorphism becomes $p \mapsto p+\tau$. 
10.2. Pencils of quadrics in $\mathbb{P}^{\mathbf{3}}$ and $\mathbb{P}_{\text {Skly }}^{3}$. A generic pencil of quadrics in $\mathbb{P}^{3}$ has exactly four singular members. Its base locus is a quartic elliptic curve. The smooth quadrics have two rulings on them, and the singular ones have only one ruling. The lines on the quadrics are the secant lines to the base locus.

The pencil of commutative quadrics in $\mathbb{P}\left(S_{1}^{*}\right)$ containing $E$ may be labelled as $Y_{z}, z \in E / \pm \cong \mathbb{P}^{1}$, in such a way that $Y_{z}$ is the union of the secant lines $\overline{p q}$ such that $p+q=z$. It follows that $Y_{z}=Y_{-z}$ and the four singular quadrics are $Y_{\omega}$, $\omega \in E_{2}$, the 2-torsion subgroup of $E$. When $z \notin E_{2}$, the two rulings on $Y_{z}$ are given by $\{\overline{p q} \mid p+q=z\}$ and $\{\overline{p q} \mid p+q=-z\}$.

As we now explain, the Sklyanin quadrics behave in a similar way.

The center of $S$ contains two linearly independent homogeneous elements, $\Omega_{1}$ and $\Omega_{2}$, of degree two. These give rise to a pencil of quotients $A=S /(\Omega), \Omega$ a non-zero linear combination of $\Omega_{1}$ and $\Omega_{2}$, and hence a pencil of noncommutative quadric hypersurfaces Proj $A \subset \mathbb{P}_{\text {Skly }}^{3}$. Each $A$ is a Gorenstein domain with dualizing module $\omega_{A} \cong A(-2)$ as a one-sided $A$-module.

As $S /\left(\Omega_{1}, \Omega_{2}\right)$ is a twisted homogeneous coordinate ring of $E$, Proj $S /\left(\Omega_{1}, \Omega_{2}\right)$ presents $E$ as a closed subspace of $\mathbb{P}_{\text {Skly }}^{3}$. It is the base locus of the pencil of noncommutative quadrics.

10.3. The following rule sets up a bijection between the line modules for $S$ and the secant lines to $E$ in $\mathbb{P}\left(S_{1}^{*}\right)$ : if $p, q \in E$, and $W \subset S_{1}$ is the subspace of linear forms vanishing on the $\overline{p q}$, then $S / S W$ is a line module that we denote by $L(\overline{p q})$ [12].

If $z \in E$, there is a non-zero linear combination $\Omega(z)$ of $\Omega_{1}$ and $\Omega_{2}$ such that

$$
\Omega(z) \cdot L(\overline{p q})=0 \Longleftrightarrow p+q=z \text { or } p+q=-z-2 \tau
$$

(see [12], Sect. 6). We label the noncommutative quadrics in $\mathbb{P}_{\text {Skly }}^{3}$ by

$$
Q_{z}:=\operatorname{Proj} S /(\Omega(z)), \quad z \in E .
$$

Thus $Q_{z}=Q_{-z-2 \tau}$.

10.4. Families of lines. If $z \notin E_{2}+\tau$, we say there are two families of line modules for $A$ giving "lines" on $Q_{z}$, namely $\{L(\overline{p q}) \mid p+q=z\}$ and $\{L(\overline{p q}) \mid p+q=$ $-z-2 \tau\}$.

The degree two divisors $(p)+(q)$ such that $p+q=z$ are parametrized by the points in the fiber over $z$ of the addition map $S^{2} E \rightarrow E$. These fibers are isomorphic to $\mathbb{P}^{1}$, which is why we say these lines form a family.

The next result shows that these "families" coincide with the "rulings" defined in Section 7.

Proposition 10.1. Let $L$ and $L^{\prime}$ be line modules for $A$. Then $L$ and $L^{\prime}$ belong to the same ruling if and only if they belong to the same family. 
Proof. Let $Q_{z}=\operatorname{Proj} A$. Suppose that $L=L(\overline{p q})$ and $L^{\prime}=L\left(\overline{p^{\prime} q^{\prime}}\right)$, where $p+q, p^{\prime}+q^{\prime} \in\{z,-z-2 \tau\}$.

$\Longleftarrow$ : Suppose that $p+q=p^{\prime}+q^{\prime}$. There are points $r, s \in E$ such that $p, q$, $r$, and $s$ span a secant plane, say that given by $a=0$ for $0 \neq a \in A_{1}$, and $p^{\prime}, q^{\prime}, r$, and $s$ also span a secant plane, say that given by $b=0$ for $0 \neq b \in A_{1}$.

Set $L^{\prime \prime}=L(\overline{r-\tau, s-\tau})$. By the argument in the proof of [19], Lemma 4.5, there are exact sequences

$$
0 \rightarrow L^{\prime \prime}(-1) \rightarrow A / A a \rightarrow L \rightarrow 0
$$

and

$$
0 \rightarrow L^{\prime \prime}(-1) \rightarrow A / A b \rightarrow L^{\prime} \rightarrow 0 .
$$

By Proposition 7.4, $L$ and $L^{\prime \prime}$ belong to different rulings, and so do $L^{\prime}$ and $L^{\prime \prime}$; hence $L$ and $L^{\prime}$ belong to the same ruling.

$\Longrightarrow$ : Suppose that $p+q \neq p^{\prime}+q^{\prime}$. In this case $p+q+\left(p^{\prime}+\tau\right)+\left(q^{\prime}+\tau\right)=0$, so $p, q, p^{\prime}+\tau, q^{\prime}+\tau$ span a secant plane. By [19], Lemma 4.5 , there is an exact sequence of the form $0 \rightarrow L^{\prime}(-1) \rightarrow A / x A \rightarrow L \rightarrow 0$, so $L$ and $L^{\prime}$ belong to the same ruling by Proposition 7.4.

Theorem 10.2. The Sklyanin quadric $Q_{z}$ is smooth if and only if $z+\tau \notin E_{2}$. The four singular quadrics are $Q_{\omega-\tau}, \omega \in E_{2}$.

Proof. If $z+\tau \notin E_{2}$, then $Q_{z}=Q_{-z-2 \tau}$ has two families of line modules, namely $L(\overline{p q})$ such that $p+q=z$ and $p+q=-z-2 \tau$, whereas if $z+\tau \in E_{2}$, there is only one family of line modules for $Q_{z}$, namely $L(\overline{p q})$ such that $p+q=z=-z-2 \tau$. Now by Theorem 10.1, there are two rulings on $Q_{z}$ if and only if $z+\tau \notin E_{2}$, so the result follows from Theorem 5.6.

10.5. Singular quadrics in a pencil. There is one significant way in which the pencil of Sklyanin quadrics differs from a generic pencil of quadrics in $\mathbb{P}^{3}$.

The singular locus of a singular quadric $Q$ belonging to a generic pencil in $\mathbb{P}^{3}$ is a point, and that point lies on all the lines on $Q$. However, the results in [19] (see also [15], Sect. 10) show there is no analogous result for the Sklyanin quadrics. For simplicity, we will explain this only when $\tau$ has infinite order.

When $\tau$ has infinite order the closed points in $\mathbb{P}_{\text {Skly }}^{3}$ consist of those on $E$ and a discrete family that may be labelled as

$$
\left\{p_{\omega+i \tau} \mid \omega \in E_{2}, i \in \mathbb{N}\right\}
$$

in such a way that

(a) $p_{\omega+i \tau}$ lies on the noncommutative secant line $\overline{p q}$ if and only if $p+q=\omega+i \tau$, and 
(b) if $\mathcal{F}_{\omega+i \tau} \in$ Qcoh $Q$ is the simple module corresponding to $p_{\omega+i \tau}$, then

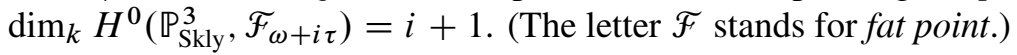

Thus, all the lines in one of the two rulings on the smooth quadrics $Q_{\omega+i \tau}=$ $Q_{\omega-(i+2) \tau}, i \in \mathbb{N}$, pass through a common point. The lines on a singular quadric $Q_{\omega-\tau}$ do not pass through a common point.

Let $i \in \mathbb{N}$ and $\omega \in E_{2}$. By [19], Sect. 4, if $p+q=\omega+i \tau$, there is an exact sequence

$$
0 \rightarrow \mathcal{O} \overline{p-(i+1) \tau, q-(i+1) \tau}(-i) \rightarrow \mathcal{O}_{\overline{p q}} \rightarrow \mathcal{F}_{\omega+i \tau} \rightarrow 0
$$

of $Q_{\omega+i \tau}$-modules; because $(p-(i+1) \tau)+(q-(i+1) \tau) \neq p+q$, the two lines in (10.1) belong to different rulings; it also follows from (10.1) that the class of $\mathcal{F}_{\omega+i \tau}$ in $K_{0}\left(Q_{\omega+i \tau}\right)$ is

$$
\left[\mathcal{F}_{\omega+i \tau}\right]=\ell-\ell^{\prime} t^{i+1}=\ell-\ell^{\prime}+(i+1) p .
$$

This shows that the positive cone of $K_{0}\left(Q_{\omega+i \tau}\right)$ is not the same as that of $K_{0}\left(\mathbb{P}^{1} \times \mathbb{P}^{1}\right)$. A computation in $K_{0}\left(Q_{\omega+i \tau}\right)$ using Proposition 9.3 gives

$$
\left(\mathcal{F}_{\omega+i \tau}, \mathcal{F}_{\omega+i \tau}\right)=2,
$$

so $p_{\omega+i \tau}$ behaves like a curve with self-intersection -2 .

10.6. Similar behavior is exhibited by the primitive quotient rings of the enveloping algebra of $\mathfrak{s l}(2, \mathbb{C})$ (cf. [9], [21] and [23]). More precisely, the homogenized enveloping algebra of $\mathfrak{s} l(2, \mathbb{C})$ is the coordinate ring of a quantum $\mathbb{P}^{3}$ that contains a pencil of noncommutative quadrics and those noncommutative quadrics behave like the Sklyanin quadrics. In particular, the finite dimensional irreducible representations of $\mathfrak{s}(2, \mathbb{C})$ provide points on certain of these quadrics that also behave like -2-curves - they have self-intersection -2 .

10.7. The quadrics in a generic pencil in $\mathbb{P}^{3}$ can be viewed as the fibers of a family $X \rightarrow \mathbb{P}^{1}$. The total space $X \subset \mathbb{P}^{3} \times \mathbb{P}^{1}$ is smooth. It seems likely that the analogous noncommutative 3-fold $X_{n c} \subset \mathbb{P}_{\text {Skly }}^{3} \times \mathbb{P}^{1}$ is also smooth, but we do not know how to tackle this problem.

10.8. Our methods apply to the pencil of noncommutative quadrics in the noncommutative $\mathbb{P}^{3}$ associated to the enveloping algebra of $\mathfrak{s}(2, \mathbb{C})$. This pencil of noncommutative quadrics is analogous to the commutative pencil of quadrics generated by a double plane $w^{2}=0$ and $x^{2}+y^{2}+z^{2}=0$. The noncommutative pencil contains a "double plane" and one more singular noncommutative quadric that corresponds to the unique primitive quotient of $U(\mathfrak{s l}(2, \mathbb{C}))$ having infinite global dimension. That particular quotient of $U(\mathfrak{s}(2, \mathbb{C}))$ is a simple ring, so has no finite dimensional simple module; this is analogous to the fact that the singular Sklyanin quadrics are not the ones having a point that causes infinite global dimension. The homological properties of the quotients of $U\left(\mathfrak{s} \mathfrak{l}_{2}\right)$ are described in [21]. 
10.9. Let $Q$ be a smooth noncommutative quadric surface. It would be interesting to show that there is a map $Q \rightarrow \mathbb{P}^{1}$ in the sense of [17], Defn. 2.3, to define and study the fibers of such a map, and to show that $Q$ is the disjoint union of these fibers in a suitable sense. It would also be interesting to examine quadric hypersurfaces in noncommutative analogues of $\mathbb{P}^{n}$ for $n>3$.

\section{References}

[1] M. Artin, J. Tate, and M. Van den Bergh, Modules over regular algebras of dimension 3. Invent. Math. 106 (1991), 335-388. Zbl 0763.14001 MR 1128218

[2] M. Artin and J. J. Zhang, Noncommutative projective schemes. Adv. Math. 109 (1994), 228-287. Zbl 0833.14002 MR 1304753

[3] A. Beilinson, V. Ginzburg, and W. Soergel, Koszul duality patterns in representation theory. J. Amer. Math. Soc. 9 (1996), 473-527. Zbl 0864.17006 MR 1322847

[4] R.-O. Buchweitz, Maximal Cohen-Macaulay modules and Tate cohomology over Gorenstein rings. Unpublished manuscript, University of Hannover, Germany, 1986. Available online at http://hdl.handle.net/1807/16682

[5] R.-O. Buchweitz, D. Eisenbud, and J. Herzog, Cohen-Macaulay modules on quadrics. In Singularities, representation of algebras, and vector bundles (Lambrecht, 1985), Lecture Notes in Math. 1273, Springer, Berlin 1987, 58-116. Zbl 0633.13008 MR 0915169

[6] D. Eisenbud, Homological algebra on a complete intersection, with an application to group representations. Trans. Amer. Math. Soc. 260 (1980), 35-64. Zbl 0444.13006 MR 570778

[7] P. Jørgensen, Non-commutative graded homological identities. J. London Math. Soc. (2) 57 (1998), 336-350. Zbl 0922.16025 MR 1644217

[8] P. Jørgensen, Linear free resolutions over non-commutative algebras. Compos. Math. 140 (2004), 1053-1058. Zbl 1073.16007 MR 2059230

[9] L. Le Bruyn and S. P. Smith, Homogenized sl(2). Proc. Amer. Math. Soc. 118 (1993), 725-730. Zbl 0795.16029 MR 1136235

[10] L. Le Bruyn, S. P. Smith, and M. Van den Bergh, Central extensions of three-dimensional Artin-Schelter regular algebras. Math. Z. 222 (1996), 171-212. Zbl 0876.17019 MR 1429334

[11] T. Levasseur, Some properties of non-commutative regular graded rings. Glasgow Math. J. 34 (1992), 277-300. Zbl 0824.16032 MR 1181768

[12] T. Levasseur and S. P. Smith, Modules over the 4-dimensional Sklyanin algebra. Bull. Soc. Math. France 121 (1993), 35-90. Zbl 0823.17020 MR 1207244

[13] B. Shelton and M. Vancliff, Embedding a quantum rank three quadric in a quantum $\mathbf{P}^{3}$. Comm. Algebra 27 (1999), 2877-2904. Zbl 0936.16023 MR 1687269

[14] E. K. Sklyanin, Some algebraic structures connected with the Yang-Baxter equation. Funktsional. Anal. i Prilozhen. 16 (1982), no. 4, 27-34; English transl. Funct. Anal. Appl. 16 (1983), 263-270. Zbl 0513.58028 MR 684124 
[15] S. P. Smith, The four-dimensional Sklyanin algebras. K-Theory 8 (1994), 65-80. Zbl 0809.16051 MR 1273836

[16] S. P. Smith, Some finite-dimensional algebras related to elliptic curves. In Representation theory of algebras and related topics (Mexico City, 1994), CMS Conf. Proc. 19, Amer. Math. Soc., Providence, RI, 1996, 315-348. Zbl 0856.16009 MR 1388568

[17] S. P. Smith, Subspaces of non-commutative spaces. Trans. Amer. Math. Soc. 354 (2002), 2131-2171. Zbl 0998.14003 MR 1885647

[18] S. P. Smith and J. T. Stafford, Regularity of the four-dimensional Sklyanin algebra. Compositio Math. 83 (1992), 259-289. Zbl 0758.16001 MR 1175941

[19] S. P. Smith and J. M. Staniszkis, Irreducible representations of the 4-dimensional Sklyanin algebra at points of infinite order. J. Algebra 160 (1993), 57-86. Zbl 0809.16052 MR 1237078

[20] S. P. Smith and M. Van den Bergh, A new Koszul duality. In preparation.

[21] J. T. Stafford, Homological properties of the enveloping algebra $U\left(\mathrm{Sl}_{2}\right)$. Math. Proc. Cambridge Philos. Soc. 91 (1982), 29-37. Zbl 0478.17006 MR 633253

[22] J. Tate and M. Van den Bergh, Homological properties of Sklyanin algebras. Invent. Math. 124 (1996), 619-647. Zbl 0876.17010 MR 1369430

[23] M. Van den Bergh, A translation principle for the four-dimensional Sklyanin algebras. $J$. Algebra 184 (1996), 435-490. Zbl 0876.17011 MR 1409223

[24] M. Van den Bergh, Existence theorems for dualizing complexes over non-commutative graded and filtered rings. J. Algebra 195 (1997), 662-679. Zbl 0894.16020 MR 1469646

[25] M. Van den Bergh, Blowing up of non-commutative smooth surfaces. Mem. Amer. Math. Soc. 154 (2001), no. 734. Zbl 0998.14002 MR 1846352

[26] A. Yekutieli and J. J. Zhang, Serre duality for noncommutative projective schemes. Proc. Amer. Math. Soc. 125 (1997), 697-707. Zbl 0860.14001 MR 1372045

Received August 8, 2011

S. P. Smith, Department of Mathematics, Box 354350, University of Washington, Seattle, WA 98195-4350, U.S.A.

E-mail: smith@math.washington.edu

M. Van den Bergh, Departement WNI, Limburgs Universitair Centrum, Universitaire Campus, Building D, 3590 Diepenbeek, Belgium

E-mail: michel.vandenbergh@luc.ac.be 